\title{
Risk measures for processes and BSDEs
}

\author{
Irina Penner* \\ Humboldt-Universität zu Berlin \\ Unter den Linden 6, 10099, Berlin, Germany \\ Anthony Réveillac ${ }^{\dagger}$ \\ Université Paris-Dauphine \\ CEREMADE UMR CNRS 7534 \\ Place du Maréchal De Lattre De Tassigny \\ 75775 Paris cedex 16 France
}

July 27,2021

\begin{abstract}
The paper analyzes risk assessment for cash flows in continuous time using the notion of convex risk measures for processes. By combining a decomposition result for optional measures, and a dual representation of a convex risk measure for bounded càdlàg processes, we show that this framework provides a systematic approach to the both issues of model ambiguity, and uncertainty about the time value of money. We also establish a link between risk measures for processes and BSDEs.
\end{abstract}

Key words: Convex risk measures for processes, Discounting ambiguity, Model ambiguity, Cash subadditivity, Decomposition of optional measures, BSDEs.

AMS 2010 subject classification: Primary: 60G07; Secondary: 91B30, 91B16, 60H10, 60G40.

JEL subject classification: D81.

\section{Introduction}

Classical risk assessment methods in Mathematical Finance focus on uncertain payoffs, that are described by random variables on some probability space. In this context, the payments are usually assumed to be discounted, and their timing does not matter for the risk evaluation beyond that. However, the assumption that time value of money can be resolved by a simple discounting procedure is too restrictive in many situations. The purpose of the present paper is to provide a risk assessment method in continuous time, that accounts not only for model ambiguity, but also for uncertainty about time value of money.

An axiomatic approach to assessing risks in Mathematical Finance was initiated in 2, 3, 20, 22 by introducing the concepts of coherent and convex monetary risk measures. One

\footnotetext{
* penner@math.hu-berlin.de

†anthony.reveillac@ceremade.dauphine.fr
} 
of the main axioms of a monetary risk measure, which distinguishes it from a classical utility functional, is cash invariance. A cash invariant risk measure computes the minimal capital requirement, that has to be added to a position in order to make it acceptable. On the other hand, as argued in [18], cash invariance is a too stringent requirement, since it postulates that future payoffs and present capital reserves are expressed in terms of the same numéraire. Therefore, while monetary risk measures provide a robust method to deal with model ambiguity, they do not allow one to deal with the issue of discounting ambiguity. To remedy this drawback, a new type of risk measures was introduced in [18, where the axiom of cash invariance is replaced by cash subadditivity.

It was noted in [1], that risk measures for processes introduced in [12, 14] provide an alternative approach to the problem of discounting uncertainty. The more flexible framework of stochastic processes allows one to relax the axiom of cash invariance without loosing the interpretation of a risk measure as a minimal capital requirement. Consequently, risk measures for processes provide a natural framework to deal with both model ambiguity, and uncertainty about time value of money. Moreover, uncertainty about time value of money has a rather general interpretation in this context: It includes interest rate ambiguity, but also robust optimal stopping problems for american type options as in [39, 8, 9. And restricted to random variables, risk measures for processes reduce to cash subadditive risk measures introduced in [18. The general structure becomes visible through the robust representation of a convex risk measure for processes given in [1, Theorem 3.8, Corollary $3.9]$ in discrete time framework. One of the main goals of the present paper is to extend this result of [1] to continuous time framework. It requires two steps: a dual representation of a monetary convex risk measure on the set of bounded càdlàg processes in terms of suitably penalized optional measures, and a decomposition of optional measures into the model and the discounting components.

The latter decomposition result is of independent mathematical interest. It provides a Fubini-type disintegration of a positive finite measure on the optional $\sigma$-field into a randomized stopping time $D$, which defines a random measure on the time-axis, and into a local martingale $L$, which can be essentially seen as a model on the underlying probability space. In discrete time, such decomposition was proved in [1, Theorem 3.4]; a continuous time version appeared independently in [27, Theorem 2.1]. In Theorem 4.2 we complement the result of [27] by providing necessary and sufficient conditions for a couple $(L, D)$ to define an optional measure. We also give a more precise statement on the uniqueness of the decomposition, and, in difference to [27], a direct proof of it.

Omitting technical details, our discussion shows that taking expectation on the optional $\sigma$-field essentially amounts to computing expectation of a discounted process on the underlying probability space. A robust representation of a risk measure for processes in terms of optional measures as in 1] seems therefore fairy natural. Mathematical precision of this idea is however technically demanding in continuous time framework, since there is no dominating measure on the optional $\sigma$-field, that would allow one to apply the usual $L^{\infty}-L^{1}$ duality as in the context of random variables. A general dual representation of a convex risk measure for bounded càdlàg processes given in [12, Theorem 3.3] involves pairs of optional and predictable measures, respectively. However, all examples given in [12, and also examples of risk measures defined by BSDEs in the present paper can be represented in terms of ordinary optional measures only. We provide therefore conditions, under which the 
representation from [12] reduces to such a simplified form.

One of the reasons for popularity of classical risk measures is their well established relation to the concepts of BSDEs and $g$-expectations in continuous time Brownian framework. The papers [35, 40, 5] were among the first to identify a solution of a BSDE with a convex driver as a time consistent dynamic risk measure. The strong notion of cash invariance in this context is reflected by the condition that the driver of the BSDE does not depend on the current level of the risk $y$. If the driver does depend on $y$ and is monotone, the solution to the corresponding BSDE becomes cash subadditive; this was noted in [18.

In the present paper we aim to establish an analogous link between risk measures for processes and BSDEs. The results of [18] suggest to consider to this end BSDEs with monotone convex drivers, which in our case should depend on the whole path of the process. Indeed, we show that a BSDE with a convex monotone generator defines a time consistent dynamic convex risk measure for processes, if the generator depends on the sum $X+Y$ of the current levels of the capital requirement $Y$, and the cumulated cash flow $X$. Moreover, one may add a reflection term to such a BSDE, ensuring that the sum $Y+X$ stays above zero. The resulting reflected BSDE still fits into the format of risk measures for processes. Whereas dependence of the driver on $Y+X$ corresponds to interest rate ambiguity, the reflection term appears in case of uncertainty about stopping times; this becomes visible in the dual representations we provide for the corresponding BSDEs.

The paper is organized as follows: After fixing setup and notation in Section 2 , and recalling basic facts about risk measures for processes in Section 3, we focus on the structure of optional measures in Section 4. This section is presented in a self-contained way, and might be read independently of the rest of the paper. The main result here is Theorem 4.2, which provides decomposition of optional measures. The predictable case is treated in Proposition 4.6, the section ends with the discussion of how one may associate a probability measure to the local martingale appearing in the decomposition. Section 5 deals with duality theory for bounded càdlàg processes. Section 6 combines the results of Sections 4 and 5 by providing a general robust representation of a monetary convex risk measure for processes; Section 17 is devoted to BSDEs. Some technical results used in Section 7 are proved in the Appendix.

\section{Preliminaries and notation}

In this paper we consider a filtered probability space $\left(\Omega, \mathcal{F}_{T},\left(\mathcal{F}_{t}\right)_{t \in[0, T]}, \mathbb{P}\right)$ satisfying usual conditions. The time horizon $T$ is a fixed number in $[0, \infty]$. For $T=\infty$ we assume that $\mathcal{F}_{T}=$ $\sigma\left(\cup_{t \in[0, \infty)} \mathcal{F}_{t}\right)$. We denote by $\mathcal{O}$ (respectively by $\mathcal{P}$ ) the optional (respectively predictable) $\sigma$-field with respect to $\left(\mathcal{F}_{t}\right)_{t \in[0, T]}$. For any $\mathcal{F}_{T} \times[0, T]$ measurable process $X$ we denote by ${ }^{o} X$ (respectively ${ }^{p} X$ ) its optional (respectively predictable) projection.

We use càdlàg versions of any (local) martingales. For an adapted càdlàg process $X$ we denote by $X^{c}$ the continuous part of $X$, and by $\Delta_{\tau} X$ the jump of $X$ at a stopping time $\tau$ with $0 \leq \tau \leq T$, i.e., $\Delta X_{\tau}:=X_{\tau}-X_{\tau-}$. $\operatorname{Var}(X)$ denotes the variation of $X,[X]$ the quadratic variation, and $\langle X\rangle$ the continuous part of quadratic variation, as long as these processes are well defined. For any two adapted càdlàg processes $X$ and $Y$ we write $X \leq Y$, if $X_{t} \leq Y_{t}$ for all $t \mathbb{P}$-a.s.. 
As usually, $\int_{t}$ denotes the (stochastic) integral over $(t, \cdot]$. If the lower bound $t$ should be included into the integration area, we use the notation $\int_{[t, .]}$.

By $\mathcal{R}^{\infty}$ we denote the set of all adapted càdlàg processes $X$ that are essentially bounded, i.e., such that

$$
\|X\|_{\mathcal{R}^{\infty}}:=\left\|X^{*}\right\|_{L^{\infty}}<\infty, \quad \text { where } \quad X^{*}:=\sup _{0 \leq t \leq T}\left|X_{t}\right|
$$

\section{Convex risk measures for processes}

The notion of monetary convex risk measures for processes, that we use in this paper, was introduced in [12. It was also studied in [13, [14, 11. In this section we recall definitions and some basic results from these papers.

A process $X \in \mathcal{R}^{\infty}$ should be understood in our framework as a value process, which models the evolution of some financial value. It can also be seen as a cumulated cash flow. For instance, the process $m \mathbf{1}_{[t, T]}$ describes a single payment of $m$ amounts of cash at time $t \leq T$. This interpretation is in line with the axiom of cash invariance in the next definition.

Definition 3.1. A map $\rho: \mathcal{R}^{\infty} \rightarrow \mathbb{R}$ is called a monetary convex risk measure for processes if it satisfies the following properties:

- Cash invariance: for all $m \in \mathbb{R}$,

$$
\rho\left(X+m \mathbf{1}_{[0, T]}\right)=\rho(X)-m ;
$$

- (Inverse) Monotonicity: $\rho(X) \geq \rho(Y)$ if $X \leq Y$;

- Convexity: for all $\lambda \in[0,1]$,

$$
\rho(\lambda X+(1-\lambda) Y) \leq \lambda \rho(X)+(1-\lambda) \rho(Y)
$$

- Normalization: $\rho(0)=0$.

A convex risk measure is called a coherent risk measure for processes if it has in addition the following property for all $X \in \mathcal{R}^{\infty}$ :

- Positive homogeneity: for all $\lambda \in \mathbb{R}$ with $\lambda \geq 0$,

$$
\rho(\lambda X)=\lambda \rho(X) .
$$

Remark 3.2. If $\rho$ is a monetary convex risk measure for processes, the functional $\phi:=-\rho$ defines a monetary or money based utility functional, which is sometimes alternatively used in the literature.

Remarks 3.3. 1. The axioms of inverse monotonicity and convexity in Definition [3.1 go back to the classical utility theory, and have obvious interpretations. Normalization is assumed merely for notational convenience, any convex risk measure $\tilde{\rho}$ with $\tilde{\rho}(0) \in \mathbb{R}$ can be normalized by passing to $\rho:=\tilde{\rho}-\tilde{\rho}(0)$. 
2. Cash invariance gives rise to the monetary interpretation of a risk measure as follows: We define the acceptance set of a monetary convex risk measure as

$$
\mathcal{A}:=\left\{X \in \mathcal{R}^{\infty} \mid \rho(X) \leq 0\right\} .
$$

By convexity and monotonicity the set $\mathcal{A}$ is convex and solid. Cash invariance yields the following representation of a risk measure:

$$
\rho(X)=\inf \left\{m \in \mathbb{R} \mid X+m \mathbf{1}_{[0, T]} \in \mathcal{A}\right\} .
$$

In other words, $\rho(X)$ is the minimal capital requirement, that has to be added to the process $X$ at time 0 in order to make it acceptable. Conversely, a functional defined by (3.1) for a given convex solid set $\mathcal{A}$ is a (not necessarily normalized) monetary convex risk measure for processes.

In difference to a monetary risk measure for random variables, cf., e.g., [21, Definition 4.1], the axiom of cash invariance in Definition 3.1 specifies the timing of the cash flow: Only payments made at the same time as the risk assessment shift it in a linear way. This makes risk measures for processes sensitive to the timing of the payment, and establishes a conceptional difference to the more common notion of risk measures for random variables. Even if restricted to random variables, i.e., to processes of the form $X \mathbf{1}_{[T]}$ for some $X \in$ $L^{\infty}\left(\Omega, \mathcal{F}_{T}, \mathbb{P}\right)$, a risk measure in the sense of Definition 3.1 does not reduce to a risk measure in the sense of [21, Definition 4.1]. This aspect was noted in [1, Section 5], and it can be made precise using the notion of cash subadditivity.

Definition 3.4. A convex risk measure for processes $\rho$ is called

- cash subadditive, if for all $t \geq 0$ and $m \in \mathbb{R}$

$$
\begin{aligned}
& \rho\left(X+m 1_{[t, T]}\right) \geq \rho(X)-m \text { for } m \geq 0 \\
&(\text { resp. } \leq \text { for } m \leq 0) ;
\end{aligned}
$$

- cash additive at $t$ for some $t>0$, if

$$
\rho\left(X+m 1_{[t, T]}\right)=\rho(X)-m, \quad \forall m \in \mathbb{R} ;
$$

- cash additive, if it is cash additive at all $t \in[0, T]$.

The notion of cash subadditivity was introduced by El Karoui and Ravanelli [18] in the context of risk measures for random variables. It appears naturally in the context of risk measures for processes, as noted in [1, Proposition 5.2].

Proposition 3.5. Every convex risk measure for processes is cash subadditive.

Proof. Follows directly from monotonicity and cash invariance.

Due to cash subadditivity property, risk measures for processes provide a more flexible framework than risk measures for random variables. They allow to capture not only model uncertainty, but also uncertainty about the time value of money. This will be made precise in Section [6, and requires two steps: The first step consists in providing a dual representation of a monetary convex risk measure on $\mathcal{R}^{\infty}$ in terms of suitably penalized optional measures. In the second step, optional measures will be decomposed into state price deflators, describing the model component, and randomized stopping times, describing the discounting component. We begin with the latter decomposition result for optional measures. 


\section{Decomposition of optional measures}

In this section we analyze the structure of finite positive measures $\mu$ on the optional $\sigma$-field $\mathcal{O}$, that have no mass on $\mathbb{P}$-evanescent sets. Such measures are called optional $\mathbb{P}$-measures in [16], here we simply call them optional measures.

The set of optional measures will be denoted by $\mathcal{M}(\mathcal{O})$, and the subset of optional measures $\mu$ with $\mu(\mathcal{O})=1$ by $\mathcal{M}_{1}(\mathcal{O})$. We also introduce the spaces

$$
\mathcal{B}^{1}=\left\{a=\left(a_{t}\right)_{t \in[0, T]} \mid a \text { adapted, right-continuous, of finite variation, } \operatorname{Var}(a) \in L^{1}(\mathbb{P})\right\},
$$

and the space of random measures

$$
\mathcal{B}_{+}^{1}:=\left\{a \in \mathcal{B}^{1} \mid a_{0-}:=0, a \text { non-decreasing }\right\} .
$$

Due to Doléans representation result, cf., e.g., [16, Theorem VI 65], $\mu \in \mathcal{M}(\mathcal{O})$ if and only if there exists a process $a \in \mathcal{B}_{+}^{1}$ such that

$$
\mathbb{E}_{\mu}[X]=\mathbb{E}\left[\int_{[0, T]} X_{s} d a_{s}\right]
$$

for every bounded optional process $X$. So we can (and will) identify the space $\mathcal{M}(\mathcal{O})$ with $\mathcal{B}_{+}^{1}$, and the space $\mathcal{M}_{1}(\mathcal{O})$ with

$$
\mathcal{Z}_{1}:=\left\{a \in \mathcal{B}_{+}^{1} \mid \mathbb{E}\left[a_{T}\right]=1\right\} .
$$

Next we prove an auxiliary result on extension of local martingales; we apply here terminology and results from [25, Chapter V]. For a given non-decreasing sequence of stopping times $\left(\tau_{n}\right)_{n \in \mathbb{N}}$ such that $\tau:=\lim _{n} \tau_{n}$ is a predictable stopping time, we consider a stochastic interval of the form $\cup_{n \in \mathbb{N}} \llbracket 0, \tau_{n} \rrbracket$. The interval can be either open or closed at the right boundary $\tau$ : Defining $B:=\cap_{n}\left\{\tau_{n}<\tau\right\}$, we have that $\cup_{n \in \mathbb{N}} \llbracket 0, \tau_{n} \rrbracket=\llbracket 0, \tau \llbracket$ on $B$, and $\cup_{n \in \mathbb{N}} \llbracket 0, \tau_{n} \rrbracket=\llbracket 0, \tau \rrbracket$ on $B^{\mathrm{c}}$. We call a process $L$ a local martingale (resp. a semimartingale, a supermartingale) on $\cup_{n \in \mathbb{N}} \llbracket 0, \tau_{n} \rrbracket$, if for any stopping time $\sigma$ such that $\llbracket 0, \sigma \rrbracket \subseteq \cup_{n \in \mathbb{N}} \llbracket 0, \tau_{n} \rrbracket$ the stopped process $L^{\sigma}$ is a local martingale (resp. a semimartingale, a supermartingale). The following lemma extends [11, Proposition 1], cf. also [7, Lemma 6.10] to non-continuous local martingales.

Lemma 4.1. Let $\left(\tau_{n}\right)_{n \in \mathbb{N}}$ be an non-decreasing sequence of stopping times, such that $\tau=$ $\lim _{n} \tau_{n}$ is a predictable stopping time. Assume further that $L$ is a nonnegative local martingale on the stochastic interval $\cup_{n \in \mathbb{N}} \llbracket 0, \tau_{n} \rrbracket$. Then there exists a càdlàg local martingale $\tilde{L}=\left(\tilde{L}_{t}\right)_{t \in[0, T]}$, such that $\tilde{L}=\tilde{L}^{\tau}$, and $L=\tilde{L}$ on $\cup_{n \in \mathbb{N}} \llbracket 0, \tau_{n} \rrbracket$.

Proof. We define the extension of $L$ as

$$
\tilde{L}_{t}:= \begin{cases}L_{t} & \text { on }\{t<\tau\}, \\ L_{\tau} & \text { on }\{\tau \leq t \leq T\} \cap B^{\mathrm{c}}, \\ \lim _{s \uparrow \tau, s \in \llbracket 0, \tau \llbracket \cap \mathbb{Q}} L_{s} & \text { on }\{\tau \leq t \leq T\} \cap B,\end{cases}
$$


where $B=\cap_{n}\left\{\tau_{n}<\tau\right\}$. Since $L$ is a nonnegative supermartingale on $\cup_{n \in \mathbb{N}} \llbracket 0, \tau_{n} \rrbracket$, the left limit $L_{\tau-}$ exists $\mathbb{P}$-a.s.. In particular, the process $\tilde{L}$ is well defined, $L=\tilde{L}$ on $\cup_{n \in \mathbb{N}} \llbracket 0, \tau_{n} \rrbracket$, and $\tilde{L}$ is a supermartingale on $[0, T]$ by [25, Lemma 5.17, Proposition 5.8]. In fact, $\tilde{L}$ is a local martingale. To see this, we use the Doob-Meyer decomposition of the supermartingale $\tilde{L}=\tilde{M}-\tilde{a}$, where $\tilde{M}$ is a local martingale, and $\tilde{a}$ a predictable non-decreasing process. Since $\tilde{L}$ is a local martingale on $\llbracket 0, \tau \llbracket$ and constant on $\rrbracket \tau, T \rrbracket$, uniqueness of the Doob-Meyer decomposition implies

$$
\tilde{a}_{t}= \begin{cases}0 & \text { on } t<\tau, \\ \Delta \tilde{a}_{\tau} & \text { on } \tau \leq t \leq T .\end{cases}
$$

We will show that

$$
\mathbb{E}\left[\Delta \tilde{a}_{\tau}\right]=\mathbb{E}\left[\Delta \tilde{M}_{\tau}\right]-\mathbb{E}\left[\Delta \tilde{L}_{\tau}\right]=0,
$$

which implies $\tilde{a} \equiv 0$, and proves that $\tilde{L}=\tilde{M}$ is a local martingale. In order to see (4.4), note that $\mathbb{E}\left[\Delta \tilde{M}_{\tau}\right]=0$, since $\tau$ is predictable, and $\tilde{M}$ a local martingale. Moreover, by [25, Theorem 5.3], cf. also [11, Lemma 1], there exists a non-decreasing sequence of stopping times $\left(\sigma_{n}\right)_{n \in \mathbb{N}}$, such that $\cup_{n \in \mathbb{N}} \llbracket 0, \tau_{n} \rrbracket=\cup_{n \in \mathbb{N}} \llbracket 0, \sigma_{n} \rrbracket$, and $L^{\sigma_{n}}$ is a uniformly integrable martingale for each $n$. We have $\Delta \tilde{L}_{\tau}=\lim _{n} \Delta L_{\tau}^{\sigma_{n}}$, since $\Delta \tilde{L}_{\tau}=0$ on $B$, and $\Delta \tilde{L}_{\tau}=\Delta L_{\tau}$ on $B^{\mathrm{c}}$. In addition, $\left|\Delta L_{\tau}^{\sigma_{n}}\right| \leq\left|\Delta \tilde{L}_{\tau}\right| \in L^{1}(\mathbb{P})$ for all $n \in \mathbb{N}$, since $\tilde{L}$ is a nonnegative supermartingale. Hence, dominated convergence implies

$$
\mathbb{E}\left[\Delta \tilde{L}_{\tau}\right]=\lim _{n} \mathbb{E}\left[\Delta L_{\tau}^{\sigma_{n}}\right]=0,
$$

where we have used that $\tau$ is predictable and $L^{\sigma_{n}}$ is a martingale for the second equality. This concludes the proof.

We are now ready to state the main result of this section.

Theorem 4.2. A process $a:=\left(a_{t}\right)_{t \in[0, T]}$ is an non-decreasing, right-continuous, adapted process with $a_{0-}=0$ and $\mathbb{E}\left[a_{T}\right]=1$, if and only if there exists a pair of adapted càdlàg processes $(L, D):=\left(L_{t}, D_{t}\right)_{t \in[0, T]}$, such that

1) $L$ is a non-negative local martingale with $L_{0}=1$ and $L_{T-}=\mathbb{E}\left[L_{T} \mid \mathcal{F}_{T-}\right]$;

2) $D$ is a non-increasing process with $D_{0-}=1$ and $\left\{D_{T}>0\right\} \subseteq\left\{L_{T}=0\right\}$;

3) The non-negative supermartingale $\left(L_{t} D_{t}\right)_{t \in[0, T]}$ is of class $(D)$;

4) $a_{t}=-\int_{[0, t]} L_{s} d D_{s} \quad \forall t \in[0, T]$, with the convention $a_{0}=-L_{0} \Delta D_{0}=1-D_{0}$.

The processes $L$ and $D$ are unique up to undistinguishability on $\llbracket 0, \tau \llbracket$, where

$$
\tau:=\inf \left\{t \in[0, T] \mid a_{t}=a_{T}\right\} .
$$

Moreover, the pair $(L, D)$ can be chosen such that in addition

5) $L_{t}=L_{0}+\int_{0}^{t} \mathbf{1}_{\left\{D_{s-}>0\right\}} d L_{s}, \quad D_{t}=1+\int_{0}^{t} \mathbf{1}_{\left\{L_{s}>0\right\}} d D_{s} \quad \forall t \in[0, T]$

holds. Under this condition $L$ and $D$ are essentially unique on $[0, T]$. 
Dolean's representation result, cf., e.g., [16, Theorem VI 65], implies immediately the following corollary.

Corollary 4.3. We have $\mu \in \mathcal{M}(\mathcal{O})$ if and only if there exists a pair of processes $(L, D)$, satisfying properties 1)-3) of Theorem 4.2, where in 1) $L_{0}=\mu(\mathcal{O})$, such that

$$
\mathbb{E}_{\mu}[X]=\mathbb{E}\left[-\int_{[0, T]} X_{s} L_{s} d D_{s}\right]
$$

for every bounded optional process $X$.

Before giving the proof of Theorem 4.2, let us note that a discrete time version of it appeared in [1, Theorem 3.4], and a continuous time version was proved in [27, Theorem 2.1]. Here we complement the result of [27] by providing necessary and sufficient conditions for a couple $(L, D)$ to define an optional measure. In particular, sufficiency requires property 3 ), that did not appear in [27, Theorem 2.1]. We also provide a more precise statement on the uniqueness of the couple $(L, D)$, and, in difference to [27, a direct proof of it. It involves only conditions 1), 2), and 4) of Theorem 4.2, and hence applies also to [27, Theorem 2.1].

Remark 4.4. In Theorem 4.2 we choose the process $D$ to be non-increasing, i.e., the measure $-d D$ to be positive, since in our framework $D$ is interpreted as a discounting process. One can always switch to the non-decreasing process $K:=1-D$ as in [27, Theorem 2.1], in order to have a positive measure in the representation (4.5).

Proof of Theorem 4.2. The proof will be obtained in several steps. We begin with the "only if" part.

\section{Step 1}

We consider the non-negative supermartingale $U$ defined by

$$
U_{t}:=\mathbb{E}\left[a_{T} \mid \mathcal{F}_{t}\right]-a_{t}=: M_{t}-a_{t}, \quad t \in[0, T],
$$

The process $U$ is of class (D), and it is a potential if and only if $\Delta a_{T}=0$. We define the stopping times

$$
\tau_{n}:=\inf \left\{t \in[0, T] \mid U_{t} \leq \frac{1}{n}\right\}, \quad n \in \mathbb{N},
$$

and

$$
\begin{aligned}
\tau:=\lim _{n \rightarrow \infty} \tau_{n} & =\inf \left\{t \in[0, T] \mid U_{t-}=0 \text { or } U_{t}=0\right\} \\
& =\inf \left\{t \in[0, T] \mid a_{t}=a_{T}\right\} .
\end{aligned}
$$

We have $\tau \leq T \mathbb{P}$-a.s., and $U$ vanishes on $\llbracket \tau, T \rrbracket$ by [16, Theorem VI.17].

To determine the process $D$, we set $D_{0-}:=1$, and define $\left(D_{t}\right)_{t \in[0, T]}$ as the unique solution of the SDE

$$
D_{t}=1-\int_{0}^{t} \frac{D_{s-}}{U_{s}+\Delta a_{s}} d a_{s}, \quad t \in[0, T]
$$

i.e.,

$$
D_{t}:=\exp \left(-\int_{0}^{t} \frac{1}{U_{s}} d a_{s}^{c}\right) \prod_{0 \leq s \leq t, \Delta a_{s}>0} \frac{U_{s}}{U_{s}+\Delta a_{s}}, \quad t \in[0, T] .
$$


Note that $D$ is well-defined, right-continuous, and non-increasing on $[0, T]$, with $D_{0}=1-a_{0}$, $D=D^{\tau},\left\{D_{\tau-}=0\right\} \subseteq\left\{U_{\tau-}=0\right\}$, and $\left\{D_{\tau}=0\right\} \subseteq\left\{\Delta a_{\tau}>0\right\} \cup\left\{D_{\tau-}=0\right\} \mathbb{P}$-a.s.

The process $L$ should be intuitively defined as the stochastic exponential of $\int_{0}^{\cdot} \mathbf{1}_{\left\{U_{s-}>0\right\}} \frac{1}{U_{s-}} d M_{s}$. In order to make this definition rigorous, let $A:=\left\{U_{\tau-}=0\right\}$, and denote by $\tau_{A}$ the restriction of $\tau$ to $A$, i.e.,

$$
\tau_{A}:= \begin{cases}\tau & \text { on } A \\ T & \text { otherwise }\end{cases}
$$

Note that $\tau_{A}$ is a predictable stopping time, since $\tau_{A}=\lim _{n} \tilde{\tau}_{n}$, where

$$
\tilde{\tau}_{n}:= \begin{cases}\tau_{n} & \text { on }\left\{\tau_{n}<\tau\right\} \\ T & \text { on }\left\{\tau_{n}=\tau\right\}\end{cases}
$$

Since $M=M^{\tau}$, and $\frac{1}{U_{s-}}$ is bounded on $\llbracket 0, \tilde{\tau}_{n} \rrbracket \cap \llbracket 0, \tau \rrbracket$, the stochastic integral $\int_{0}^{\cdot} \frac{1}{U_{s-}} d M_{s}$ is well defined on each $\llbracket 0, \tilde{\tau}_{n} \rrbracket$, and hence on $\cup_{n \in \mathbb{N}} \llbracket 0, \tilde{\tau}_{n} \rrbracket$. Thus we can define the process $L$ as the stochastic exponential of the local martingale $\int_{0}^{\cdot} \frac{1}{U_{s-}} d M_{s}$ on $\cup_{n \in \mathbb{N}} \llbracket 0, \tilde{\tau}_{n} \rrbracket$, i.e.

$$
L_{t}:=\exp \left(\int_{0}^{t} \frac{1}{U_{s-}} d M_{s}^{c}-\frac{1}{2} \int_{0}^{t}\left|\frac{1}{U_{s-}}\right|^{2} d\langle M\rangle_{s}\right) \times \prod_{\substack{0<s \leq t, \Delta M_{s} \neq 0}}\left(1+\frac{\Delta M_{s}}{U_{s-}}\right)
$$

for $(\omega, t) \in \cup_{n \in \mathbb{N}} \llbracket 0, \tilde{\tau}_{n} \rrbracket$. Then $L$ solves

$$
L_{t}=1+\int_{0}^{t} \frac{L_{s-}}{U_{s-}} d M_{s},
$$

and is a non-negative local martingale on $\cup_{n \in \mathbb{N}} \llbracket 0, \tilde{\tau}_{n} \rrbracket$. By Lemma 4.1, $L$ can be extended to a local martingale on $[0, T]$, which we also denote by $L$. It follows from (4.12) and from (4.3), that $L$ solves the $\mathrm{SDE}$

$$
L_{t}=1+\int_{0}^{t} \mathbf{1}_{\left\{U_{s-}>0\right\}} \frac{L_{s-}}{U_{s-}} d M_{s}, \quad t \in[0, T],
$$

and can be written as

$$
\begin{aligned}
L_{t}:=\exp \left(\int_{0}^{t \wedge \tau}\right. & \left.\frac{1}{U_{s-}} d M_{s}^{c}-\frac{1}{2} \int_{0}^{t \wedge \tau}\left|\frac{1}{U_{s-}}\right|^{2} d\langle M\rangle_{s}\right) \\
& \times \prod_{\substack{0<s \leq t \wedge \tau, \Delta M_{s} \neq 0}}\left(1+\frac{\Delta M_{s}}{U_{s-}}\right), \quad t \in[0, T] .
\end{aligned}
$$

We slightly deviate here from the usual definition of a stochastic exponential by allowing the continuous part of $L$ to become zero. Indeed, the set

$$
\left\{L_{\tau}=0\right\}=\left\{\lim _{v \uparrow \tau_{A}} \int_{0}^{v \wedge \tau_{A}}\left|\frac{1}{U_{s-}}\right|^{2} d\langle M\rangle_{s}=\infty\right\} \subseteq A
$$


might have positive probability, cf. [27, Example 2.5]. We use in (4.14) the convention $L_{t}(\omega):=0$ for $\omega \in\left\{L_{\tau}=0\right\} \cap\{\tau \leq t\}$. Note that the jump part of $L$ is well defined at $\tau$, since $\Delta M_{\tau}=0$ on $\left\{U_{\tau-}=0\right\}$.

It follows either from (4.13) or from (4.14), that

$$
\mathbb{E}\left[L_{T} \mid \mathcal{F}_{T-}\right]=\mathbb{E}\left[L_{T-}+\mathbf{1}_{\left\{U_{T-}>0\right\}} L_{T-} \frac{\Delta M_{T}}{U_{T-}} \mid \mathcal{F}_{T-}\right]=L_{T-},
$$

since $\mathbb{E}\left[\Delta M_{T} \mid \mathcal{F}_{T-}\right]=0$ both for $T<\infty$ and $T=\infty$ due to the fact that $M$ is a uniformly integrable martingale.

\section{Step 2}

We show that $D$ and $L$ provide a multiplicative decomposition of $U$, i.e.,

$$
U_{t}=L_{t} D_{t} \quad \forall t \in[0, T]
$$

First we prove this equality on $\cup_{n \in \mathbb{N}} \llbracket 0, \tilde{\tau}_{n} \rrbracket$. To this end, we note that by the same argumentation as in Step 1, the stochastic integral $\int_{0}^{\cdot} \frac{1}{U_{s-}} d U_{s}$ is well defined on $\cup_{n \in \mathbb{N}} \llbracket 0, \tilde{\tau}_{n} \rrbracket$. Thus $U$ can be written as the stochastic exponential of $\int_{0}^{\cdot} \frac{1}{U_{s-}} d U_{s}$, i.e.,

$$
U_{t}=U_{0} \exp \left(\int_{0}^{t} \frac{1}{U_{s-}} d U_{s}^{c}-\frac{1}{2} \int_{0}^{t}\left|\frac{1}{U_{s-}}\right|^{2} d\langle M\rangle_{s}\right) \times \prod_{\substack{0<s \leq t, \Delta U_{s} \neq 0}}\left(\frac{U_{s}}{U_{s-}}\right)
$$

on $\cup_{n \in \mathbb{N}} \llbracket 0, \tilde{\tau}_{n} \rrbracket$. Plugging (4.10) and (4.11) into (4.15), and noting that

$$
\prod_{\substack{0<s \leq t, \Delta U_{s} \neq 0}} \frac{U_{s}}{U_{s-}}=\prod_{\substack{0<s \leq t, 0 M_{s} \neq 0}}\left(1+\frac{\Delta M_{s}}{U_{s-}}\right) \times \prod_{\substack{0<s \leq t, \Delta a_{s} \neq 0}} \frac{U_{s}}{U_{s}+\Delta a_{s}},
$$

we obtain (4.16). It remains to prove (4.15) for $(\omega, t) \in \llbracket \tau_{A}, T \rrbracket$ and $\omega \in\left\{U_{\tau_{A^{-}}}=0\right\}$. Thanks to the existence of the left limits, we obtain

$$
0=U_{\tau_{A}-}=L_{\tau_{A}-} D_{\tau_{A}-}=L_{\tau_{A}} D_{\tau_{A}}
$$

where we have used that $\Delta D_{\tau_{A}}=\Delta L_{\tau_{A}}=0$ on $\left\{U_{\tau_{A}-}=0\right\}=\left\{U_{\tau_{-}}=0\right\}$ by definitions of $D$ and $L$. Hence,

$$
U_{t}=U_{\tau_{A}}=0=L_{\tau_{A}} D_{\tau_{A}}=L_{t} D_{t} \quad \text { for }(\omega, t) \in \llbracket \tau_{A}, T \rrbracket \text { and } \omega \in\left\{U_{\tau_{A^{-}}}=0\right\} \text {. }
$$

This concludes the proof of (4.15). Note that (4.15) implies in particular property 3 ) of the theorem, and $\left\{D_{T}>0\right\} \subseteq\left\{L_{T}=0\right\}$, since $U_{T}=0$.

\section{Step 3:}

We now prove properties 4) and 5) of the theorem. First note that 4) holds at 0 by definitions of $D$ and $L$. Hence it remains to prove

$$
a_{t}-a_{0}=-\int_{0}^{t} L_{s} d D_{s} \quad \forall t \in[0, T] .
$$


The multiplicative decomposition (4.15), integration by parts, and the SDE (4.13) yield for each $t \in[0, T]$

$$
\begin{aligned}
U_{t}=L_{t} D_{t} & =L_{0} D_{0}+\int_{0}^{t} L_{s} d D_{s}+\int_{0}^{t} D_{s-} d L_{s} \\
& =U_{0}+\int_{0}^{t} L_{s} d D_{s}+\int_{0}^{t} \mathbf{1}_{\left\{U_{s-}>0\right\}} d M_{s}
\end{aligned}
$$

Since $\left\{M_{s}>0\right\} \subseteq\left\{U_{s-}>0\right\}$, we have

$$
M_{t}-M_{0}=\int_{0}^{t} \mathbf{1}_{\left\{M_{s}>0\right\}} d M_{s}=\int_{0}^{t} \mathbf{1}_{\left\{U_{s-}>0\right\}} d M_{s}
$$

and thus

$$
\int_{0}^{t} L_{s} d D_{s}=U_{t}-U_{0}-\left(M_{t}-M_{0}\right)=-\left(a_{t}-a_{0}\right) .
$$

Concerning property 5), note that by definition of $D$ we have $\left\{U_{t-}>0\right\} \subseteq\left\{D_{t-}>0\right\}$ for all $t \in[0, T]$. Thus (4.13) implies for each $t \in[0, T]$

$$
L_{t}=L_{0}+\int_{0}^{t} \mathbf{1}_{\left\{U_{s-}>0\right\}} \frac{L_{s-}}{U_{s-}} d M_{s}=L_{0}+\int_{0}^{t} \mathbf{1}_{\left\{D_{s->0}\right\}} d L_{s}
$$

Similarly, by definition of $L$ we have $\left\{U_{t}+\Delta a_{t}>0\right\} \subseteq\left\{L_{t}>0\right\}$ for all $t \in[0, T]$, and hence (4.9) yields for each $t \in[0, T]$

$$
D_{t}=1-\int_{0}^{t} \mathbf{1}_{\left\{U_{s}+\Delta a_{s}>0\right\}} \frac{D_{s-}}{U_{s}+\Delta a_{s}} d a_{s}=1+\int_{0}^{t} \mathbf{1}_{\left\{L_{s}>0\right\}} d D_{s} .
$$

\section{Step 4:}

In order to prove uniqueness, we first show that every pair of processes $(\tilde{L}, \tilde{D})$ satisfying properties 1), 2), and 4) of Theorem 4.2 provides a multiplicative decomposition of the supermartingale $U$ defined in (4.6), that is,

$$
U_{t}=\tilde{L}_{t} \tilde{D}_{t} \quad \forall t \in[0, T]
$$

This holds clearly at $T$, since $U_{T}=0$ and $\left\{\tilde{D}_{T}>0\right\} \subseteq\left\{\tilde{L}_{T}=0\right\}$. In order to prove (4.18) on $[0, T)$, let $\left(\sigma_{n}\right)_{n \geq 1}$ be a localizing sequence for $\tilde{L}$, i.e. $\sigma_{n} \nearrow T \mathbb{P}$-a.s., and $L^{\sigma_{n}}$ is a uniformly integrable martingale for each $n$. Let further $\sigma$ be any stopping time. Then property 4) yields

$$
\begin{aligned}
1 & =\mathbb{E}\left[a_{T}\right]=\mathbb{E}\left[a_{T}-a_{\sigma \wedge \sigma_{n}}+a_{\sigma \wedge \sigma_{n}}-a_{0-}\right] \\
& =\mathbb{E}\left[a_{T}-a_{\sigma \wedge \sigma_{n}}\right]-\mathbb{E}\left[\int_{0}^{\sigma \wedge \sigma_{n}} \tilde{L}_{s} d \tilde{D}_{s}\right]-\mathbb{E}\left[L_{0} \Delta D_{0}\right] \\
& =\mathbb{E}\left[U_{\sigma \wedge \sigma_{n}}\right]-\mathbb{E}\left[\int_{0}^{\sigma \wedge \sigma_{n}} \tilde{L}_{\sigma \wedge \sigma_{n}} d \tilde{D}_{s}\right]-\mathbb{E}\left[L_{0} \Delta D_{0}\right]
\end{aligned}
$$




$$
=\mathbb{E}\left[U_{\sigma \wedge \sigma_{n}}\right]-\mathbb{E}\left[\tilde{L}_{\sigma \wedge \sigma_{n}} \tilde{D}_{\sigma \wedge \sigma_{n}}\right]+1,
$$

where we have used uniform integrability of the martingale $L^{\sigma_{n}}$, and [16, VI.57]. Hence, by [15. IV.87 b)] the processes $U^{\sigma_{n}}$ and $\tilde{L}^{\sigma_{n}} \tilde{D}^{\sigma_{n}}$ are indistinguishable for each $n$. Since $\sigma_{n} \rightarrow T$ $\mathbb{P}$-a.s., (4.18) holds on $[0, T)$.

In particular, since $\Delta a_{t}=-\tilde{L}_{t} \Delta \tilde{D}_{t}$ by 4 ), (4.18) yields $U_{t}+\Delta a_{t}=\tilde{L}_{t} \tilde{D}_{t-}$ for all $t$. This implies on $\llbracket 0, \tau \llbracket$ :

$$
\tilde{D}_{t}-\tilde{D}_{0}=\int_{0}^{t} d \tilde{D}_{s}=\int_{0}^{t} \frac{\tilde{L}_{s} \tilde{D}_{s-}}{\tilde{L}_{s} \tilde{D}_{s-}} d \tilde{D}_{s}=-\int_{0}^{t} \frac{\tilde{D}_{s-}}{U_{s}+\Delta a_{s}} d a_{s}
$$

So $\tilde{D}$ is a solution to the SDE (4.9) on $\llbracket 0, \tau \llbracket$, and thus coincides with $D$ on this set. Since $U=L D=\tilde{L} \tilde{D}$, this implies further $L=\tilde{L}$ on $\llbracket 0, \tau \llbracket$, and $L_{\tau-}=\tilde{L}_{\tau-}, D_{\tau-}=\tilde{D}_{\tau-}$. Moreover, since $L_{\tau} D_{\tau}=\tilde{L}_{\tau} \tilde{D}_{\tau}=U_{\tau}=0$, property 4 ) yields

$$
L_{\tau} D_{\tau-}=-L_{\tau} \Delta D_{\tau}=-\Delta a_{\tau}=-\tilde{L}_{\tau} \Delta \tilde{D}_{\tau}=\tilde{L}_{\tau} \tilde{D}_{\tau-} .
$$

Thus $\tilde{L}_{\tau}=L_{\tau}>0$ on $\left\{\Delta a_{\tau}>0\right\}$, and hence $\tilde{D}_{\tau}=D_{\tau}=0$ on $\left\{\Delta a_{\tau}>0\right\}$ by (4.15) and (4.18), which implies already $D=\tilde{D}$ on $\left\{\Delta a_{\tau}>0\right\}$. On $\left\{\Delta a_{\tau}=0\right\}$ we have $\tilde{D}_{\tau}=\tilde{D}_{\tau-}=$ $D_{\tau-}=D_{\tau}$, and $\tilde{L}_{\tau-}=L_{\tau-}=0$ on $\left\{\Delta a_{\tau}=0\right\} \cap\left\{D_{\tau-}>0\right\}$, since $U_{\tau-}=L_{\tau-} D_{\tau-}=0$ on $\left\{\Delta a_{\tau}=0\right\}$. Non-negativity and local martingale property imply then $L=\tilde{L}$ on $\left\{\Delta a_{\tau}=\right.$ $0\} \cap\left\{D_{\tau-}>0\right\}$.

If we assume in addition, that $(\tilde{L}, \tilde{D})$ satisfies property 5$)$ of the theorem, we obtain also $\tilde{L}=\tilde{L}^{\tau}=L^{\tau}=L$ on $\left\{\Delta a_{\tau}>0\right\} \cup\left\{D_{\tau-}=0\right\}$, and $\tilde{D}=\tilde{D}^{\tau}=D^{\tau}=D$ on $\left\{\Delta a_{\tau}=\right.$ $0\} \cap\left\{D_{\tau-}>0\right\}$, which proves equality (in the sense of undistinguishability) on $[0, T]$.

\section{Step 5:}

We now proof the "if" part of the theorem. Obviously, any two processes $L$ and $D$ satisfying properties 1) and 2) define a non-decreasing, right-continuous, adapted process $a$ via 4). It remains to prove that $\mathbb{E}\left[a_{T}\right]=1$. To this end let $\left(\sigma_{n}\right)_{n \in \mathbb{N}}$ be a localizing sequence for $L$. Note that w.l.o.g. we can assume that $\sigma_{n}<T$ for all $n$, otherwise we switch to $\sigma_{n} \wedge\left(T-\frac{1}{n}\right)$ in case $T<\infty$. Using 1), 2), 4), uniformly integrability of the martingale $L^{\sigma_{n}}$, and [16, VI.57] we obtain for each $n \in \mathbb{N}$ :

$$
\begin{aligned}
\mathbb{E}\left[a_{\sigma_{n}}\right] & =\mathbb{E}\left[-\int_{\left[0, \sigma_{n}\right]} L_{t \wedge \sigma_{n}} d D_{t}\right]=\mathbb{E}\left[-\int_{\left[0, \sigma_{n}\right]} L_{\sigma_{n}} d D_{t}\right] \\
& =\mathbb{E}\left[-L_{\sigma_{n}} D_{\sigma_{n}}+L_{0} D_{0-}\right]=\mathbb{E}\left[-L_{\sigma_{n}} D_{\sigma_{n}}\right]+1
\end{aligned}
$$

By monotone convergence, $\mathbb{E}\left[a_{\sigma_{n}}\right] \rightarrow \mathbb{E}\left[a_{T-}\right]$ with $n \rightarrow \infty$, and $\mathbb{E}\left[L_{\sigma_{n}} D_{\sigma_{n}}\right] \rightarrow \mathbb{E}\left[L_{T-} D_{T-}\right]$, since $L D$ is of class (D). Moreover,

$$
\mathbb{E}\left[L_{T-} D_{T-}\right]=\mathbb{E}\left[L_{T} D_{T-}\right]=\mathbb{E}\left[-L_{T} \Delta D_{T}\right]=\mathbb{E}\left[\Delta a_{T}\right],
$$

where we have used $L_{T-}=\mathbb{E}\left[L_{T} \mid \mathcal{F}_{T-}\right], D_{T}=0$ on $\left\{L_{T}>0\right\}$ and 4 ). Hence (4.19) implies

$$
\mathbb{E}\left[a_{T}\right]=\mathbb{E}\left[a_{T-}\right]+\mathbb{E}\left[\Delta a_{T}\right]=1 .
$$


Remarks 4.5. 1. Our proof of Theorem 4.2 is based on the idea that any pair of processes $(L, D)$ satisfying conditions 1$)$-3) provides a multiplicative decomposition of the supermartingale $U$ defined in (4.6). The construction of $L$ and $D$ is inspired by the classical multiplicative decomposition results as in [24], [25, Theorem 6.17]. However, in difference to these results, the non-increasing process $D$ in our case is in general not predictable, even if the corresponding process a is. As it can be seen from (4.10), $D$ is predictable, if a is predictable, and it does not jump at the same time as the martingale $M$; cf. also Remark 4.7 later on in text.

2. In [1, Theorem 3.4], which is a discrete time counterpart of Theorem 4.2, the nonincreasing process $D$ is predictable. However, this is just a matter of notation: The process D appearing in [1, Theorem 3.4] corresponds to the predictable process $D_{-}$of Theorem 4.2. Indeed, if $(L, D)$ is a couple of processes as in Theorem 4.2, and if we can associate a measure $Q$ on $(\Omega, \mathcal{F})$ to the local martingale $L$, as explained later on in text, representation (4.5) takes the form

$$
\mathbb{E}_{\mu}[X]=\mathbb{E}_{Q}\left[\int_{[0, T]} D_{s-} d X_{s}\right]
$$

for any bounded semimartingale $X$ with $X_{0-}:=0$. This representation corresponds to (3.8) of [1, Theorem 3.4].

Clearly, Theorem 4.2 provides for any predictable process $a$ a decomposition $(L, D)$, such that $\int_{0} L_{t} d D_{t}$ is predictable. However, if one seeks to construct a predictable process $a$ starting with a couple $(L, D)$, it requires more conditions than 1)-3) of Theorem 4.2 to ensure predictability. In this case, $D$ should "compensate" the non-predictable jumps of the local martingale $L$, i.e., the jump process $\left(\sum_{s \leq t} L_{s} \Delta D_{s}\right)_{t}$ should be predictable. This additional assumption is not very handy. In the predictable case it seems more natural to use a different construction, namely $a=\int L_{-} d D$ with a predictable process $D$ and a local martingale $L$. This is done in the next proposition.

Proposition 4.6. A process $a:=\left(a_{t}\right)_{t \in[0, T]}$ is an non-decreasing, right-continuous, predictable process with $a_{0-}=0$ and $\mathbb{E}\left[a_{T}\right]=1$, if and only if there exists a pair of adapted càdlàg processes $(L, D)$, satisfying properties 1)-3) of Theorem 4.2. such that in addition $D$ is predictable, and

$$
\text { 4') } a_{t}=-\int_{[0, t]} L_{s-} d D_{s} \quad \forall t \in[0, T] \text { with the convention } L_{0-}:=1 \text { holds. }
$$

The processes $L$ and $D$ are unique up to undistinguishability on $\llbracket 0, \tau \llbracket$, where $\tau$ is as in Theorem 4.2. Moreover, the pair $(L, D)$ can be chosen such that in addition

$$
\left.5^{\prime}\right) L_{t}=L_{0}+\int_{0}^{t} \mathbf{1}_{\left\{D_{s}>0\right\}} d L_{s}, \quad D_{t}=1+\int_{0}^{t} \mathbf{1}_{\left\{L_{s-}>0\right\}} d D_{s} \quad \forall t \in[0, T]
$$

holds. Under this condition $L$ and $D$ are essentially unique on $[0, T]$.

Proof. The proof of the "if" part follows exactly as in Step 5 of the proof of Theorem 4.2 , Obviously, the process $a$ defined by $4^{\prime}$ ) is predictable, and, since $D$ is predictable, the equality (4.19) holds in the same way for $\mathbb{E}\left[-\int_{\left[0, \sigma_{n}\right]} L_{t \wedge \sigma_{n}-} d D_{t}\right]$. 
To prove "only if", we use the classical multiplicative decomposition of the supermartingale $U$ defined in (4.6) as

$$
U_{t}=\mathbb{E}\left[a_{T} \mid \mathcal{F}_{t}\right]-a_{t}=M_{t}-a_{t}, \quad t \in[0, T] .
$$

The construction of $D$ and $L$ basically follows as in the proof on Theorem 4.2, with the difference that $U_{-}$has to be replaced by the predictable projection of $U$, denoted by ${ }^{p} U$. The process $D$ is defined via

$$
D_{t}=1-\int_{0}^{t} \frac{D_{s-}}{{ }^{p} U_{s}} d a_{s}, \quad t \in[0, T],
$$

i.e., $D_{0-}:=1$ and

$$
D_{t}:=\exp \left(-\int_{0}^{t} \frac{1}{{ }^{p} U_{s}} d a_{s}^{c}\right) \prod_{0 \leq s \leq t, \Delta a_{s}>0} \frac{{ }^{p} U_{s}}{U_{s-}}, \quad t \in[0, T] .
$$

$D$ is well-defined, predictable, right-continuous, and non-increasing on $[0, T]$. We have also $D=D^{\tau}$, where $\tau$ is the stopping time defined in (4.8).

To define the process $L$, let $B:=\left\{{ }^{p} U_{\tau}=0\right\}$, and denote by $\tau_{B}$ the restriction of $\tau$ to $B$. Due to [25. (6.23), (6.24), Corollary 6.28], there exists an non-decreasing sequence of stopping times $\left(\sigma_{n}\right)$, such that $\frac{1}{p_{U}} \mathbf{1}_{\llbracket 0, \sigma_{n} \rrbracket} \leq n$ for all $n \in \mathbb{N}, \tau=\lim _{n} \sigma_{n}$, and

$$
\cup_{n} \llbracket 0, \sigma_{n} \rrbracket=\cup_{n} \llbracket 0, \tau_{n} \rrbracket \cap \llbracket 0, \tau_{B} \llbracket=\llbracket 0, \tau \rrbracket \cap \llbracket 0, \tau_{B} \llbracket,
$$

where $\tau_{n}$ are stopping times defines in (4.7). Hence we have $\tau_{B}=\lim _{n} \tilde{\sigma}_{n}$, where

$$
\tilde{\sigma}_{n}:= \begin{cases}\sigma_{n} & \text { on }\left\{\sigma_{n}<\tau\right\}, \\ T & \text { on }\left\{\sigma_{n}=\tau\right\},\end{cases}
$$

and $\tau_{B}$ is a predictable stopping time.

Using the same argumentation as in Step 1 of the proof of Theorem 4.2, we define the process $L$ as the stochastic exponential of the local martingale $\int_{0}^{\cdot} \frac{1}{{ }^{p} U_{s}} d M_{s}$ on $\cup_{n \in \mathbb{N}} \llbracket 0, \tilde{\sigma}_{n} \rrbracket$, and extend it to a local martingale on $[0, T]$ as in Lemma 4.1. This yields

$$
L_{t}=1+\int_{0}^{t} \mathbf{1}_{\left\{{ }^{p} U_{s}>0\right\}} \frac{L_{s-}}{{ }^{p} U_{s}} d M_{s}, \quad t \in[0, T],
$$

and

$$
\begin{aligned}
L_{t}=\exp \left(\int_{0}^{t \wedge \tau} \frac{1}{{ }^{p} U_{s}} d M_{s}^{c}-\right. & \left.\frac{1}{2} \int_{0}^{t \wedge \tau}\left|\frac{1}{p U_{s}}\right|^{2} d\langle M\rangle_{s}\right) \\
& \times \prod_{\substack{0<s \leq t \wedge \tau, \Delta M_{s} \neq 0}}\left(\frac{U_{s}}{p U_{s}}\right), \quad t \in[0, T] .
\end{aligned}
$$

$L$ is well defined at $\tau$, since $\Delta M_{\tau}=a_{\tau}-M_{\tau-}=-{ }^{p} U_{\tau}$, and thus $\Delta M_{\tau}=0$ on $\left\{{ }^{p} U_{\tau}=0\right\}$. We also have

$$
\mathbb{E}\left[L_{T} \mid \mathcal{F}_{T-}\right]=\mathbb{E}\left[L_{T-}+\mathbf{1}_{\left\{{ }^{p} U_{T}>0\right\}} L_{T-} \frac{\Delta M_{T}}{{ }^{p} U_{T}} \mid \mathcal{F}_{T-}\right]=L_{T-} .
$$


Due to [25, Theorem 6.31], $L$ and $D$ provide a multiplicative decomposition of $U$, i.e.,

$$
U_{t}=L_{t} D_{t}
$$

holds on $\cup_{n} \llbracket 0, \sigma_{n} \rrbracket=\llbracket 0, \tau \rrbracket \cap \llbracket 0, \tau_{B} \llbracket$. Since $U=U^{\tau}, L=L^{\tau}$, and $D=D^{\tau}$, (4.22) holds also on $\cup_{n \in \mathbb{N}} \llbracket 0, \tilde{\sigma}_{n} \rrbracket$. It remains to prove (4.22) for $(\omega, t) \in \llbracket \tau_{B}, T \rrbracket$ and $\omega \in\left\{{ }^{p} U_{\tau}=0\right\}$. To this end, note that $D_{\tau_{B}}=0$ on $\left\{{ }^{p} U_{\tau}=0\right\} \cap\left\{\Delta a_{\tau_{B}}>0\right\}$ by (4.20), hence $0=U_{\tau_{B}}=L_{\tau_{B}} D_{\tau_{B}}$ on this set. On the set $\left\{{ }^{p} U_{\tau}=0\right\} \cap\left\{\Delta a_{\tau_{B}}=0\right\}$ we have ${ }^{p} U_{\tau}=U_{\tau-}$, thus $\tau_{B}=\tau_{A}$, and we can conclude as in Step 2 of the proof of Theorem 4.2 .

Thanks to (4.22) and integration by parts formula, we have

$$
U_{t}=\mathbb{E}\left[a_{T} \mid \mathcal{F}_{t}\right]-a_{t}=L_{t} D_{t}=\int_{0}^{t} D_{s} d L_{s}+\int_{[0, t]} L_{s-} d D_{s}, \quad t \in[0, T]
$$

and thus property 4') follows from the uniqueness of the Doob-Meyer decomposition. Concerning property $\left.5^{\prime}\right)$, note that by definition of $D$ we have $\left\{{ }^{p} U_{t}>0\right\} \subseteq\left\{D_{t}>0\right\}$ for all $t \in[0, T]$, and hence

$$
L_{t}=L_{0}+\int_{0}^{t} \mathbf{1}_{\left\{{ }^{p} U_{s}>0\right\}} d L_{s}=L_{0}+\int_{0}^{t} \mathbf{1}_{\left\{D_{s>0}\right\}} d L_{s} .
$$

Similarly, by definition of $L$ we have $\left\{{ }^{p} U_{t}>0\right\} \subseteq\left\{L_{t-}>0\right\}$ for all $t \in[0, T]$, thus

$$
D_{t}=1-\int_{0}^{t} \mathbf{1}_{\left\{{ }^{p} U_{s}>0\right\}} \frac{D_{s-}}{p_{U_{s}}} d a_{s}=1+\int_{0}^{t} \mathbf{1}_{\left\{L_{s-}>0\right\}} d D_{s}, \quad t \in[0, T] .
$$

In order to prove uniqueness, we can again apply the same argumentation as in Step 4 of the proof of Theorem 4.2, to conclude that every pair of processes $(\tilde{L}, \tilde{D})$ satisfying properties 1)-4') of Proposition 4.6 provides a multiplicative decomposition of the supermartingale $U$. Hence uniqueness on $\cup_{n} \llbracket 0, \sigma_{n} \rrbracket=\llbracket 0, \tau \rrbracket \cap \llbracket 0, \tau_{B} \llbracket$ follows from 25, Corollary 6.28, Theorem 6.31]. In particular, we have $L_{\tau_{-}}=\tilde{L}_{\tau_{-}}, D_{\tau_{-}}=\tilde{D}_{\tau_{-}}$, and $\tilde{L}_{\tau}=L_{\tau}=0$ on $B \cap\left\{L_{\tau_{B}-}=0\right\}$ due to the local martingale property. Moreover, since

$$
0={ }^{p} U_{\tau_{B}}=U_{\tau_{B}-}-\Delta a_{\tau_{B}}=\tilde{L}_{\tau_{B}-} \tilde{D}_{\tau_{B}-}+\tilde{L}_{\tau_{B}-} \Delta \tilde{D}_{\tau_{B}}=L_{\tau_{B}-} \tilde{D}_{\tau_{B}}
$$

on $B$, we have $\tilde{D}_{\tau}=D_{\tau}=0$ on $B \cap\left\{L_{\tau_{B}-}>0\right\}$. Property $\left.5^{\prime}\right)$ implies further $\tilde{D}_{\tau}=D_{\tau}$ on $B \cap\left\{L_{\tau_{B}-}=0\right\}, \tilde{L}_{\tau}=L_{\tau}$ on $B \cap\left\{L_{\tau_{B}-}>0\right\}$, and also $\tilde{D}=D, \tilde{L}=L$ on $\rrbracket \tau, T \rrbracket$. This concludes the proof.

Remarks 4.7. 1. If $(\underset{\tilde{L}}{L}, D)$ is the decomposition of a predictable process a as in Theorem 4.2, and $(\tilde{L}, \tilde{D})$ its decomposition as in Proposition 4.6. then $a=\int L d D=$ $\int \tilde{L}_{-} d \tilde{D}$, but in general we do not have $D=\tilde{D}$ and $L=\tilde{L}$. As it can be seen from (4.10), (4.20), (4.14), and (4.21), we have $D=\tilde{D}$ and $L=\tilde{L}$ if and only if the martingale $M=\left(\mathbb{E}\left[a_{T} \mid \mathcal{F}_{t}\right]\right)_{t \in[0, T]}$ and the process a do not jump at the same time, i.e., iff the bracket process $[M, a]=\left(\sum_{s \leq t} \Delta M_{s} \Delta a_{s}\right)_{t \in[0, T]}$ is undistinguishable from 0 .

2. In a view of the previous remark, the decompositions $(L, D)$ as in Theorem 4.2, and $(\tilde{L}, \tilde{D})$ as in Proposition 4.6 coincide if the filtration $\left(\mathcal{F}_{t}\right)$ is continuous. 
3. It follows directly from (4.10) (resp. (4.20)) and property 4) (resp. 4')), that the process a is purely discontinuous if and only if the process $D$ is purely discontinuous.

In the rest of this section we discuss how one can associate a measure $Q$ on $\left(\Omega, \mathcal{F}_{T}\right)$ to the local martingale $L$; in this case representation (4.5) takes the form

$$
\mathbb{E}_{\mu}[X]=\mathbb{E}_{Q}\left[-\int_{[0, T]} X_{s} d D_{s}\right]
$$

We fix a process $a \in \mathcal{Z}_{1}$, or alternatively, a measure $\mu \in \mathcal{M}_{1}(\mathcal{O})$, and denote by $(L, D)$ the corresponding decomposition satisfying conditions 1)-4) of Theorem 4.2. The three following cases can occur:

Case 1: $L$ is a uniformly integrable martingale. Then we can define a probability measure $Q$ on the $\sigma$-field $\mathcal{F}_{T}$ in a straightforward way by $\frac{d Q}{d P}:=L_{T}$. We have $Q \ll P$, and $D_{T}=0 Q$ a.s. Since $L$ is uniformly integrable martingale, [16, VI.57] yields for any bounded optional process $X$

$$
\mathbb{E}\left[\int_{0}^{T} X_{t} L_{t} d D_{t}\right]=\mathbb{E}\left[L_{T} \int_{0}^{T} X_{t} d D_{t}\right]=\mathbb{E}_{Q}\left[\int_{0}^{T} X_{t} d D_{t}\right]
$$

Remark 4.8. Case 1 holds in particular, if the measure $\mu$ is concentrated on $\Omega \times\{T\}$, i.e., if $a_{t}=0$ for all $t \in[0, T)$. Then the supermartingale $U$ defined in (4.6) coincides with the uniformly integrable martingale $\left(\mathbb{E}\left[a_{T} \mid \mathcal{F}_{t}\right]\right)$ on $[0, T)$, and it's multiplicative decomposition is given by $L_{t}:=\mathbb{E}\left[a_{T} \mid \mathcal{F}_{t}\right], t \in[0, T]$, and $D_{t}:=1, t \in[0, T), D_{T}:=0$. In this case (4.5) takes the form

$$
\mathbb{E}_{\mu}[X]=\mathbb{E}_{Q}\left[X_{T}\right]
$$

Case 2: $L$ is a true martingale on $[0, T)$, which is not uniformly integrable, i.e. $\mathbb{E}\left[L_{T}\right]<1$. Note that this case can occur also if $T<\infty$, cf. [28, Remark 1.3]. In this case one can associate a measure $Q$ to the process $L$, if the filtration satisfies some additional technical conditions: Assume that $\mathcal{F}_{T}=\mathcal{F}_{T-}=\bigvee_{t \in[0, T)} \mathcal{F}_{t}$, and that $\left(\mathcal{F}_{t}\right)_{t \in[0, T)}$ is the so called $N$ augmentation of some filtered probability space, as defined in [33, Proposition 2.4], see also [10. Moreover, assume that the non-augmented filtered probability space satisfies condition $(P)$ of [33, Definition 4.1], cf. also [34].

The main idea in this case is to use Parthasarathy's (34) measure extension result, as done in [19]; see also [33, Corollary 4.10], [1, Theorem 3.4], [28, Theorem 1.1]. We define a measure $Q_{t}$ locally on each $\mathcal{F}_{t}$ by $\frac{d Q_{t}}{d \mathbb{P}}:=L_{t}$. Under the assumptions above, the consistent family $\left(Q_{t}\right)_{t \in[0, T)}$ can be extended to a unique measure $Q$ on $\mathcal{F}_{T}$, such that $\left.Q\right|_{\mathcal{F}_{t}}=Q_{t}$ for all $t$. Note that $Q$ is locally absolutely continuous with respect to $\mathbb{P}$, i.e., $Q \ll \mathbb{P}$ on each $\mathcal{F}_{t}$, $t \in[0, T)$, but $Q$ is not absolutely continuous with respect to $\mathbb{P}$ on $\mathcal{F}_{T}$. For this reason the filtration $\left(\mathcal{F}_{t}\right)$ cannot be completed with zero sets of $\mathcal{F}_{T}$. However, in this case the "usual conditions" can be replaced by $N$-usual conditions, cf. [33] and [10].

Corollary 4.9. Assume that $\mathcal{F}_{T}=\mathcal{F}_{T-}$, and that $\left(\Omega,\left(\mathcal{F}_{t}\right)_{t \in[0, T)}, \mathbb{P}\right)$ is the $N$-augmentation of a filtered probability space that satisfies the property $(P)$. Let $a \in \mathcal{Z}_{1}$ with the decomposition $(L, D)$ as in Theorem 4.2, such that the process $L$ is a martingale on $[0, T)$. Then there exists a probability measure $Q$ on $\mathcal{F}_{T}$, that is locally absolutely continuous with respect to $\mathbb{P}$, 
such that $D_{T}=0$ Q-a.s. and

$$
\mathbb{E}\left[\int_{[0, T]} X_{t} d a_{t}\right]=\mathbb{E}_{Q}\left[-\int_{[0, T]} X_{t} d D_{t}\right]
$$

for any bounded optional process $X$.

Proof. We define the measure $Q$ as explained above. Let $\tau_{n}$ be any sequence of stopping times such that $\tau_{n}<T, \tau_{n} \nearrow T P$-a.s.. Then $L^{\tau_{n}}$ is a uniformly integrable martingale for each $n$, and the same argumentation as in (4.24) yields for any bounded optional process $X$

$$
\mathbb{E}\left[\int_{0}^{\tau_{n}} X_{t} L_{t} d D_{t}\right]=\mathbb{E}_{Q}\left[\int_{0}^{\tau_{n}} X_{t} d D_{t}\right], \quad n \in \mathbb{N} .
$$

By dominated convergence, $\mathbb{E}\left[\int_{0}^{T-} X_{t} L_{t} d D_{t}\right]=\mathbb{E}_{Q}\left[\int_{0}^{T-} X_{t} d D_{t}\right]$, and it remains to prove the equality at $T$. To this end we argue as in [1]: By [26, Lemma 2, Lemma 3], the limit $L_{T-}=\lim _{t \rightarrow T} L_{t}$ exists $\mathbb{P}$ - and $Q$-a.s., and the measure $Q$ has Lebesgue decomposition on $\mathcal{F}_{T}$ with respect to $\mathbb{P}$ given by

$$
Q[A]=\int_{A} L_{T-} d \mathbb{P}+Q\left[A \cap\left\{L_{T-}=\infty\right\}\right], \quad A \in \mathcal{F}_{T} .
$$

Moreover, since $\left(D_{t}\right)_{t \in[0, T)}$ is non-increasing under $Q$, the limit $D_{T-}$ exists also $Q$-a.s. By construction, the random variable $D_{T}$ is defined under $\mathbb{P}$, and hence under $Q$ only on the set $\left\{L_{T-}<\infty\right\}$. We define $D_{T}:=0$ on the set $\left\{L_{T-}=\infty\right\}$. Note further that $L_{T}=L_{T-}$ $\mathbb{P}$-a.s., since $\mathcal{F}_{T}=\mathcal{F}_{T-}$ and $\mathbb{E}\left[L_{T} \mid \mathcal{F}_{T-}\right]=L_{T-}$. This implies

$$
Q\left[\left\{D_{T}>0\right\}\right]=\mathbb{E}_{\mathbb{P}}\left[\mathbf{1}_{\left\{D_{T}>0\right\}} L_{T-}\right]+Q\left[\left\{D_{T}>0\right\} \cap\left\{L_{T-}=\infty\right\}\right]=\mathbb{E}_{\mathbb{P}}\left[\mathbf{1}_{\left\{D_{T}>0\right\}} L_{T}\right]=0,
$$

where we have used that $L_{T} D_{T}=0 \mathbb{P}$-a.s.. Moreover, we have $D_{T-}=0$ on $\left\{L_{T-}=\infty\right\}$ $Q$-a.s. thanks to (4.26) and the fact that $L D$ is of class (D). Indeed, we have for any sequence of stopping times $\left(\tau_{n}\right)$ as above

$$
\begin{aligned}
\mathbb{E}_{Q}\left[D_{T-1} \mathbf{1}_{\left\{L_{T-}=\infty\right\}}\right] & =\mathbb{E}_{Q}\left[D_{T-}\right]-\mathbb{E}_{\mathbb{P}}\left[L_{T-} D_{T-}\right] \\
& =\mathbb{E}_{Q}\left[D_{T-}\right]-\lim _{n} \mathbb{E}_{\mathbb{P}}\left[L_{\tau_{n}} D_{\tau_{n}}\right] \\
& =\mathbb{E}_{Q}\left[D_{T-}\right]-\lim _{n} \mathbb{E}_{Q}\left[D_{\tau_{n}}\right]=0,
\end{aligned}
$$

where the the last equality holds due to monotone convergence. Hence we obtain

$$
\begin{aligned}
\mathbb{E}_{\mathbb{P}}\left[X_{T} L_{T} \Delta D_{T}\right] & =\mathbb{E}_{\mathbb{P}}\left[-X_{T} L_{T-} D_{T-}\right] \\
& =\mathbb{E}_{Q}\left[-X_{T} D_{T-}\right]-\mathbb{E}_{Q}\left[-X_{T} D_{T-} \mathbf{1}_{\left\{L_{T-}=\infty\right\}}\right] \\
& =\mathbb{E}_{Q}\left[X_{T} \Delta D_{T}\right],
\end{aligned}
$$

where we have used that $L_{T}=L_{T-}, L_{T} D_{T}=0 \mathbb{P}$-a.s., (4.26), $D_{T-}=0$ on $\left\{L_{T-}=\infty\right\}$, and $D_{T}=0 Q$-a.s.. This proves (4.25) also at $T$ and completes the proof. 
Remark 4.10. Note that $L D$ is of class $(D)$ under $\mathbb{P}$ if and only if $D_{T-}=0$ on $\left\{L_{T-}=\infty\right\}$ Q-a.s.. Indeed, the "only if" part was proved in (4.27). To see that also the converse is true, we define the stopping times

$$
\sigma_{n}:=\inf \left\{t \mid L_{t} \geq n\right\}, \quad n \in \mathbb{N} .
$$

By monotone convergence

$$
0=\mathbb{E}_{Q}\left[D_{T-} \boldsymbol{1}_{\left\{L_{T-}=\infty\right\}}\right]=\lim _{n} \mathbb{E}_{Q}\left[D_{\sigma_{n}} \boldsymbol{1}_{\left\{\sigma_{n}<T\right\}}\right]=\lim _{n} \mathbb{E}_{\mathbb{P}}\left[L_{\sigma_{n}} D_{\sigma_{n}} \mathbf{1}_{\left\{\sigma_{n}<T\right\}}\right] .
$$

Since $0 \leq D \leq 1 \mathbb{P}$-a.s., [16, Theorem VI.25] implies that LD is of class (D).

Case 3: If $L$ is a strict local martingale, and the filtration $\left(\mathcal{F}_{t}\right)_{t \in[0, T)}$ is a standard system (cf. [34, [19]), it is still possible to associate a measure $Q$ to $L$, as done in [19], cf. also [28, Theorem 1.8]. However, in this case not even the $N$-augmentation of the filtration can be used, and one would have to work with a non-completed filtration. This imposes many technical restrictions, and goes beyond the scope of the present paper.

\section{Robust representation of convex risk measures on $\mathcal{R}^{\infty}$}

In this section we first recall some notation and the representation result for convex risk measures on $\mathcal{R}^{\infty}$ from [12. We consider the space of pairs of finite variation processes

$$
\begin{aligned}
\mathcal{A}^{1}:=\left\{a:[0, T] \times \Omega \rightarrow \mathbb{R}^{2} \mid\right. & a=\left(a^{\mathrm{op}}, a^{\mathrm{pr}}\right)=\left(a_{t}^{\mathrm{op}}, a_{t}^{\mathrm{pr}}\right)_{t \in[0, T]}, \\
& a^{\mathrm{op}}, a^{\mathrm{pr}} \text { right continuous, of finite variation, } \\
& a^{\mathrm{pr}} \text { predictable, } a_{0}^{\mathrm{pr}}=0, \\
& a^{\mathrm{op}} \text { optional, purely discontinuous, } \\
& \left.\operatorname{Var}\left(a^{\mathrm{pr}}\right)+\operatorname{Var}\left(a^{\mathrm{op}}\right) \in L^{1}(\mathbb{P})\right\} .
\end{aligned}
$$

The space $\mathcal{A}^{1}$ is a Banach space with the norm

$$
\|a\|_{\mathcal{A}^{1}}:=\mathbb{E}\left[\operatorname{Var}\left(a^{\mathrm{pr}}\right)+\operatorname{Var}\left(a^{\mathrm{op}}\right)\right],
$$

and any element of $\mathcal{A}^{1}$ defines a linear form on $\mathcal{R}^{\infty}$ via

$$
a(X):=\mathbb{E}\left[\int_{0}^{T} X_{t-} d a_{t}^{\mathrm{pr}}+\int_{[0, T]} X_{t} d a_{t}^{\mathrm{op}}\right], \quad X \in \mathcal{R}^{\infty} .
$$

Let further $\mathcal{A}_{+}^{1}$ denote the subset of all non-decreasing elements of $\mathcal{A}^{1}$, and

$$
\mathcal{Z}_{1}^{d}:=\left\{a=\left(a^{\mathrm{pr}}, a^{\mathrm{op}}\right) \in \mathcal{A}_{+}^{1} \mid\|a\|_{\mathcal{A}^{1}}=1\right\} .
$$

Given a subset $\hat{\mathcal{Z}}$ of $\mathcal{Z}_{1}^{d}$, a function $\gamma: \mathcal{Z}_{1}^{d} \rightarrow[0, \infty]$ is called a penalty function on $\hat{\mathcal{Z}}$, if

$$
\inf _{a \in \hat{\mathcal{Z}}} \gamma(a)=0 \text {. }
$$


For a monetary convex risk measure for processes $\rho$, a typical penalty function is the conjugate of $\rho$ :

$$
\alpha(a):=\rho^{*}(a):=\sup _{X \in \mathcal{R}^{\infty}}(a(-X)-\rho(X))=\sup _{X \in \mathcal{A}} a(-X), \quad a \in \mathcal{Z}_{1}^{d} .
$$

Here $\mathcal{A}$ denotes the acceptance set defined in Remark 3.3 .

As usually, dual representation of a convex risk measure is closely related to its continuity properties.

Definition 5.1. A monetary convex risk measure for processes $\rho$ is called

- continuous from above with respect to sup-convergence in probability (resp. with respect to pointwise convergence in probability), if

$$
\lim _{n \rightarrow \infty} \rho\left(X^{n}\right)=\rho(X)
$$

for every non-increasing sequence $\left(X^{n}\right) \subset \mathcal{R}^{\infty}$ and $X \in \mathcal{R}^{\infty}$, such that $\left(X^{n}-X\right)^{*} \rightarrow 0$ in probability (resp. such that $X_{t}^{n}-X_{t} \rightarrow 0 \mathbb{P}$-a.s. for all $t \in[0, T]$ ).

- continuous from below with respect to sup-convergence in probability (resp. with respect to pointwise convergence in probability), if

$$
\lim _{n \rightarrow \infty} \rho\left(X^{n}\right)=\rho(X)
$$

for every non-decreasing sequence $\left(X^{n}\right) \subset \mathcal{R}^{\infty}$ and $X \in \mathcal{R}^{\infty}$, such that $\left(X_{t}^{n}-X_{t}\right)^{*} \rightarrow 0$ in probability (resp. such that $X_{t}^{n}-X_{t} \rightarrow 0 \mathbb{P}$-a.s. for all $t \in[0, T]$ ).

The following result was proved in [12, Theorem 3.3].

Theorem 5.2. For a functional $\rho$ on $\mathcal{R}^{\infty}$ the following conditions are equivalent:

1. $\rho$ can be represented as

$$
\rho(X)=\sup _{a \in \mathcal{Z}_{1}^{d}}(a(-X)-\gamma(a)), \quad X \in \mathcal{R}^{\infty},
$$

with a penalty function $\gamma$ on $\mathcal{Z}_{1}^{d}$.

2. $\rho$ is a monetary convex risk measure that is continuous from above with respect to sup-convergence in probability.

Moreover, if (1)-(2) are satisfied, the function $\alpha$ defined in (5.2) is a penalty function on $\mathcal{Z}_{1}^{d}$ such that

$$
\alpha(a) \leq \gamma(a) \text { for all } a \in \mathcal{Z}_{1}^{d},
$$

and the representation (5.3) holds also with $\gamma$ replaced by $\alpha$. 
For any $a=\left(a^{\mathrm{pr}}, a^{\mathrm{op}}\right) \in \mathcal{A}^{1}$, the linear form (5.1) can be written as

$$
\begin{aligned}
a(X)=E\left[\int_{(0, T]} X_{t-} d a_{t}^{\mathrm{pr}}+\int_{[0, T]} X_{t} d a_{t}^{\mathrm{op}}\right] \\
=E\left[\int_{[0, T]} X_{t} d\left(a_{t}^{\mathrm{pr}}+a_{t}^{\mathrm{op}}\right)-\sum_{0<t \leq T} p(\Delta X)_{t} \Delta a_{t}^{\mathrm{pr}}\right],
\end{aligned}
$$

where ${ }^{p}(\Delta X)$ denotes the predictable projection of the purely discontinuous part of $X \in \mathcal{R}^{\infty}$. For $a \in \mathcal{Z}_{1}^{d}$, the process $a^{\mathrm{pr}}+a^{\text {op }}$ defines a normalized optional measure as we have considered in Section 4, cf. (4.2). However, the linear form in (5.4) involves an additional singular term $\sum^{p}(\Delta X) \Delta a^{\mathrm{pr}}$, depending on the nature of the jumps of $X$.

Our main goal in the rest of this section will be finding conditions on the risk measure $\rho$, under which it can be represented in terms of ordinary optional measures, as defined in (4.2). This simplified form is particularly useful for construction of risk measures for processes, e.g., all examples in [12, Section 5], and also our examples in Section 7 are of this form. We begin by noting that the space of optional measures $\mathcal{B}^{1}$ defined in Section 4 can be identified with a subspace of $\mathcal{A}^{1}$.

Remark 5.3. To any $a \in \mathcal{B}^{1}$ we can associate a pair $\tilde{a}:=\left(a^{\mathrm{c}}, a-a^{\mathrm{c}}\right) \in \mathcal{A}^{1}$, where $a^{\mathrm{c}}$ denotes the continuous part of $a$, and $a-a^{\mathrm{c}}$ its purely discontinuous part. Then $\|\tilde{a}\|_{\mathcal{A}^{1}}=\mathbb{E}[\operatorname{Var}(a)]$, and

$$
\tilde{a}(X)=\mathbb{E}\left[\int_{[0, T]} X_{t} d a_{t}\right] .
$$

Conversely, any pair of processes $\tilde{a}=\left(a^{\mathrm{pr}}, a^{\mathrm{op}}\right) \in \mathcal{A}^{1}$ such that $a^{\mathrm{pr}}$ is continuous, defines an element $a:=a^{\mathrm{pr}}+a^{\mathrm{op}} \in \mathcal{B}^{1}$ such that (5.5) holds. Thus we can identify $\mathcal{B}^{1}$ with the subspace

$$
\left\{\tilde{a}=\left(a^{\mathrm{pr}}, a^{\mathrm{op}}\right) \in \mathcal{A}^{1} \mid a^{\mathrm{pr}} \text { continuous }\right\}
$$

of $\mathcal{A}^{1}$, and for any $a \in \mathcal{B}^{1}$ the linear form $a(X)$ takes the form (5.5) on $\mathcal{R}^{\infty}$.

The key to the dual representation of a convex risk measure is an appropriate continuity property. The reason why a pair of processes appears in the robust representation (5.3) is condition of continuity from above with respect to sup-convergence in probability. By [16. Lemma VII 2], sup-convergence for càdlàg functions amounts to pointwise convergence of the paths and of their left limits. Thus any positive linear functional on $\mathcal{R}^{\infty}$, that is continuous from above with respect to sup-convergence in probability, is of the form (5.4), and involves two processes of finite variation, cf. [16, Theorem VII 2].

On the other hand, by Daniell-Stone Integration Theorem (cf., e.g., [21, Theorem A.49]), any positive linear functional on $\mathcal{R}^{\infty}$, that is continuous from above with respect to pointwise convergence in probability, can be represented as in (5.5) for some $a \in \mathcal{B}_{+}^{1}$. This suggests to make a stronger requirement of continuity from above with respect to pointwise convergence in probability, in order to obtain a representation of a risk measure in terms of $\mathcal{Z}_{1}$. The requirement is necessary:

Lemma 5.4. Let $\rho$ be a functional on $\mathcal{R}^{\infty}$ such that 
1. $\rho$ can be represented as

$$
\rho(X)=\sup _{a \in \mathcal{Z}_{1}}(a(-X)-\gamma(a)), \quad X \in \mathcal{R}^{\infty},
$$

with a penalty function $\gamma$ on $\mathcal{Z}_{1}$.

Then

2. $\rho$ is a monetary convex risk measure, that is continuous from above with respect to pointwise convergence in probability.

Proof. It is easy to see that $\rho$ satisfies the axioms of Definition 3.1. Continuity from above follows by standard arguments as, e.g., in the proof [21, Lemma 4.21].

We conjecture, that conditions 1) and 2) of Lemma 5.4 are in fact equivalent. Unfortunately, after spending quite some time thinking about it, we are neither able to prove that 2 ) implies $1)$, nor could we find a counterexample.

We could prove representation (5.6) under the assumption of continuity from below with respect to pointwise convergence in probability. This is a stronger requirement than continuity from above, as shown in the next lemma. The result of this lemma is well known in the context of convex risk measures for bounded random variables, cf., e.g., 21, Remark 4.25]. However, the proof there relies on the particular representation of a risk measure for random variables, and cannot be applied in our present framework. The following general argument was communicated to us by Michael Kupper, and we thank him for allowing us to include it in this paper.

Lemma 5.5. Let $\mathcal{X}$ be a topological vector space, and $\rho: \mathcal{X} \rightarrow \mathbb{R}$ any convex functional such that $\rho(X) \leq \rho(Y)$ for any $X, Y \in \mathcal{X}$ with $Y \leq X$. Assume further that $\rho$ is continuous from below in the following sense:

$$
\rho\left(X_{n}\right) \searrow \rho(X) \text { for any non-decreasing sequence }\left(X_{n}\right) \subset \mathcal{X}, X_{n} \nearrow X .
$$

Then $\rho$ is continuous from above, i.e.,

$$
\rho\left(X_{n}\right) \nearrow \rho(X) \text { for any non-increasing sequence }\left(X_{n}\right) \subset \mathcal{X}, X_{n} \searrow X .
$$

Proof. W.l.o.g. we can assume that $\rho(0)=0$, otherwise consider $\tilde{\rho}(\cdot):=\rho(\cdot)-\rho(0)$.

First we show that continuity from below at 0 implies continuity from above at 0 . Indeed, let $\left(X_{n}\right) \subset \mathcal{X}, X_{n} \searrow 0$. Then monotonicity, convexity, $\rho(0)=0$, and continuity from below at 0 imply

$$
0 \geq \rho\left(X_{n}\right) \geq-\rho\left(-X_{n}\right) \nearrow 0 .
$$

For the general case, let $\left(X_{n}\right) \subset \mathcal{X}, X_{n} \searrow X_{0}$, and consider the functional

$$
\tilde{\rho}(X):=\rho\left(X+X_{0}\right)-\rho\left(X_{0}\right), \quad X \in \mathcal{X} .
$$

It is easy to see that $\tilde{\rho}$ is a monotone convex functional with $\tilde{\rho}(0)=0$, continuous from below in 0 . By the previous argument $\tilde{\rho}$ is continuous from above at 0 , which implies

$$
\rho\left(X_{n}\right) \nearrow \rho\left(X_{0}\right)
$$

i.e., $\rho$ is continuous from above. 
Continuity from below with respect to sup-convergence in probability for convex risk measures on $\mathcal{R}^{\infty}$ was characterized in [4, Theorem 3.1]. The following theorem combines this result with the argumentation inspired by [21, Theorem 4.22].

Theorem 5.6. Let $\rho$ be a monetary convex risk measure on $\mathcal{R}^{\infty}$, that is continuous from below with respect to pointwise convergence in probability. Then $\rho$ has representation (5.3), where any penalty function $\gamma$ is concentrated on the set $\mathcal{Z}_{1}$ of normalized optional measures. In particular, $\rho$ has the representation (5.6), and the supremum is attained, i.e., we have

$$
\rho(X)=\max _{a \in \mathcal{Z}_{1}}(a(-X)-\gamma(a)), \quad X \in \mathcal{R}^{\infty} .
$$

Moreover, the level sets

$$
\Lambda_{c}:=\left\{a \in \mathcal{Z}_{1}^{d} \mid \alpha(a) \leq c\right\}, \quad c>0,
$$

are compact in $\sigma\left(\mathcal{B}^{1}, \mathcal{R}^{\infty}\right)$.

For the proof we will use the following lemma, which is a reformulation of 21, Lemma 4.23 ], and can be proved in completely analogous way in our present context.

Lemma 5.7. Let $\rho$ be a monetary convex risk measure on $\mathcal{R}^{\infty}$ with the representation (5.3), and consider the level sets $\Lambda_{c}$ defined in (5.8). Then for any sequence $\left(X_{n}\right)$ in $\mathcal{R}^{\infty}$ such that $0 \leq X_{n} \leq 1$, the following two conditions are equivalent:

$$
\begin{aligned}
& \text { 1. } \rho\left(\lambda X_{n}\right) \rightarrow \rho\left(\lambda \boldsymbol{1}_{[0, T]}\right) \text { for each } \lambda \geq 1 \text {. } \\
& \text { 2. } \inf _{a \in \Lambda_{c}} a\left(X_{n}\right) \rightarrow 1 \text { for all } c>0 \text {. }
\end{aligned}
$$

Proof of Theorem [5.6. First we note that by Lemma 5.5] $\rho$ is continuous from above with respect to pointwise convergence in probability, hence also with respect to sup-convergence in probability, and by Theorem $5.2 \rho$ has representation (5.3) with some penalty function $\gamma$ on $\mathcal{Z}_{1}^{d}$. We will show that $\gamma(a)<\infty$ implies $a \in \mathcal{Z}_{1}$. It suffices to prove this for the minimal penalty function $\alpha$.

To this end let $\left(Y^{n}\right)_{n \in \mathbb{N}}$ be a sequence in $\mathcal{R}^{\infty}$ such that $Y_{t}^{n} \searrow 0 \mathbb{P}$-a.s. for all $t$, and consider $X^{n}:=\mathbf{1}_{[0, T]}-\delta Y^{n}$, where $\delta>0$ is chosen such that $X_{t}^{n} \geq 0$ for all $t$ (e.g. $\delta:=\frac{1}{\left\|Y^{0}\right\|_{\mathcal{R}^{\infty}+1}}$ does the job). Then $0 \leq X^{n} \leq 1$, and $\lambda X^{n} \nearrow \lambda \mathbf{1}_{[0, T]} \mathbb{P}$-a.s. for all $t$ for any $\lambda>0$. Continuity from below implies $\rho\left(\lambda X^{n}\right) \searrow \rho\left(\lambda \mathbf{1}_{[0, T]}\right)$, and by Lemma 5.7

$$
1-\delta a\left(Y^{n}\right)=a\left(X^{n}\right) \rightarrow 1 \quad \text { for all } \quad a \in \Lambda_{c} .
$$

Hence $a\left(Y^{n}\right) \searrow 0$ for all $a \in \Lambda_{c}$. i.e., $a$ is continuous from above with respect to pointwise convergence in probability. By Daniell-Stone Integration Theorem (cf., e.g., [21, Theorem A.49], [15, Theorem III 35]), there exists a positive measure $\mu$ on $(\Omega, \mathcal{O})$ such that $a(X)=\int X d \mu$ for all $X \in \mathcal{R}^{\infty}$. As in the proof of [16, Theorem VII 2], it can be seen that $\mu$ disappears on $\mathbb{P}$-evanescent sets. Then, due to Dolean's representation result [16, Theorem VI 65], and uniqueness of the linear form (5.1), we can identify $a$ with some $\tilde{a} \in \mathcal{B}_{+}^{1}$ as in Remark 5.3. This proves (with some abuse of notation) that $a \in \mathcal{B}^{1} \cap \mathcal{Z}_{1}^{d}=\mathcal{Z}_{1}$ for any $a \in \mathcal{Z}_{1}^{d}$ such that $\alpha(a)<\infty$. In particular, representation (5.6) holds. Moreover, since 
$\rho$ is continuous from below with respect to sup-convergence in probability, [4, Theorem 3.1] implies that the supremum in (5.3) is attained for each $X \in \mathcal{R}^{\infty}$ by some $\bar{a} \in \mathcal{Z}_{1}^{d}$. We must have $\gamma(\bar{a})<\infty$ in this case, and thus $\bar{a} \in \mathcal{Z}_{1}$. Compactness of the sets $\Lambda_{c}$ for any $c>0$ in $\sigma\left(\mathcal{B}^{1}, \mathcal{R}^{\infty}\right)$ follows also from [4, Theorem 3.1].

\section{Model and discounting ambiguity}

This section combines the results of Sections 4 and 5 . We denote by $\mathcal{L}_{+}$the set of all nonnegative càdlàg local martingales $L=\left(L_{t}\right)_{t \in[0, T]}$ such that $L_{T-}=\mathbb{E}\left[L_{T} \mid \mathcal{F}_{T-}\right]$, and by $\mathcal{L}_{+}^{1}$ the set of all $L \in \mathcal{L}_{+}$with $L_{0}=1$. For $L \in \mathcal{L}_{+}, \mathcal{D}(L)$ denotes the set of all processes $D$ satisfying conditions 2)-3) of Theorem 4.2, i.e.,

$$
\begin{array}{r}
\mathcal{D}(L):=\left\{D=\left(D_{t}\right)_{t \in[0, T]} \mid D \text { adapted, right-continuous, non-increasing, s.t. } D_{0-}=1,\right. \\
\left.\left\{D_{T}>0\right\} \subseteq\left\{L_{T}=0\right\}, \text { and } L D \text { is of class (D) }\right\} .
\end{array}
$$

Correspondingly, $\mathcal{D}^{\text {pr }}(L)$ denotes the set of all predictable processes as in Proposition 4.6 without jump at 0 , i.e.,

$$
\mathcal{D}^{\text {pr }}(L):=\left\{D \in \mathcal{D}(L) \mid D \text { predictable, } D_{0}=1\right\}
$$

and $\mathcal{D}^{\mathrm{d}}(L)$ the set of all $D \in \mathcal{D}(L)$ such that $D$ is a purely discontinuous process. We also introduce the set

$$
\mathcal{S}_{+}^{1}:=\left\{\left(L, D, L^{\prime}, D^{\prime}\right) \mid L, L^{\prime} \in \mathcal{L}_{+}, L_{0}+L_{0}^{\prime}=1, D \in \mathcal{D}^{\mathrm{pr}}(L), D^{\prime} \in \mathcal{D}^{\mathrm{d}}\left(L^{\prime}\right)\right\} .
$$

By Theorem 4.2, Proposition 4.6, and 3) of Remark 4.7, we can identify the sets $\mathcal{Z}_{1}^{d}$ and $\mathcal{S}_{+}^{1}$, i.e., a process $a=\left(a^{\mathrm{pr}}, a^{\mathrm{op}}\right) \in \mathcal{Z}_{1}^{d}$, iff there exist $\left(L, D, L^{\prime}, D^{\prime}\right) \in \mathcal{S}_{+}^{1}$, such that $a^{\mathrm{pr}}=$ $\int_{0}^{+} L_{s-} d D_{s}, a^{\text {op }}=\int_{[0, \cdot]} L_{s}^{\prime} d D_{s}^{\prime}$. We also deliberately identify penalty functions $\gamma$ on $\mathcal{Z}_{1}^{d}$ and on $\mathcal{S}_{+}^{1}$ via

$$
\gamma\left(L, D, L^{\prime}, D^{\prime}\right):=\gamma\left(\int_{0}^{\cdot} L_{s-} d D_{s}, \int_{[0, \cdot]} L_{s}^{\prime} d D_{s}^{\prime}\right) \quad \text { for }\left(L, D, L^{\prime}, D^{\prime}\right) \in \mathcal{S}_{+}^{1} .
$$

Combining Theorem 5.2 with Theorem 4.2 and Proposition 4.6, we obtain the following corollary.

Corollary 6.1. For a functional $\rho$ on $\mathcal{R}^{\infty}$ the following conditions are equivalent:

1. For each $X \in \mathcal{R}^{\infty}$ we have

$$
\rho(X)=\sup _{\left(L, D, L^{\prime}, D^{\prime}\right) \in \mathcal{S}_{+}^{1}}\left(\mathbb{E}\left[-\int_{0}^{T} X_{t-} L_{t-} d D_{t}-\int_{[0, T]} X_{t} L_{t}^{\prime} d D_{t}^{\prime}\right]-\gamma\left(L, D, L^{\prime}, D^{\prime}\right)\right)
$$

with a penalty function $\gamma$ on $\mathcal{S}_{+}^{1}$. 
2. $\rho$ is a monetary convex risk measure that is continuous from above with respect to sup-convergence in probability.

Thanks to Theorem 5.6, dual representation takes a simpler form under the assumption of continuity from below with respect to pointwise convergence in probability:

Corollary 6.2. If $\rho$ is a monetary convex risk measure on $\mathcal{R}^{\infty}$ that is continuous from below with respect to pointwise convergence in probability, it has the representation

$$
\rho(X)=\sup _{L \in \mathcal{L}_{+}^{1}} \sup _{D \in \mathcal{D}(L)}\left(\mathbb{E}\left[\int_{[0, T]} X_{t} L_{t} d D_{t}\right]-\gamma(L, D)\right), \quad X \in \mathcal{R}^{\infty},
$$

where

$$
\gamma(L, D):=\gamma\left(\int_{[0, \cdot]} L_{s} d D_{s}\right)
$$

is a penalty function on $\mathcal{Z}_{1}$. Moreover, the supremum in (6.2) is attained by some $L \in \mathcal{L}_{+}^{1}$ and $D \in \mathcal{D}(L)$ for each $X \in \mathcal{R}^{\infty}$.

The local martingales $L$ and $L^{\prime}$ in the representation (6.1) play the roles of state price deflators, whereas the predictable non-increasing processes $D_{-}$and $D_{-}^{\prime}$ define discounting processes for this deflators, cf. 2) of Remark 4.5. In difference to (5.3) and (5.6), representations (6.2) and (6.1) make visible the roles of model ambiguity, as described by local martingales, and of discounting ambiguity, as described by corresponding non-increasing processes. In addition, a risk measure with representation (6.1), that does not reduce to (6.2), distinguishes between inaccessible and predictable jumps of the cumulated cash flow.

Appearance of discounting processes in the representations (6.1) and (6.2) reflects cash subadditivity of the risk measure, whereas cash additivity at time $s>t$ implies that there is no discounting between $t$ and $s$ in all relevant models. This was noted in [1, Corollary 5.10, Proposition 5.11], and is extended to our present framework by the next proposition.

Proposition 6.3. Let $\rho$ be a convex risk measure for processes with representation (6.1). Then it is cash additive at time $s \in(0, T]$ if and only if

$$
D_{s-}=1 \text { on }\left\{L_{s}>0\right\}, \quad \text { and } \quad D_{s-}^{\prime}=1 \text { on }\left\{L_{s}^{\prime}>0\right\} \quad \mathbb{P} \text {-a.s. }
$$

for all $\left(L, L^{\prime}, D, D^{\prime}\right) \in \mathcal{S}_{+}^{1}$ such that $\gamma\left(L, L^{\prime}, D, D^{\prime}\right)<\infty$. In this case $\rho$ admits the representation

$$
\rho(X)=\sup _{\left(L, D, L^{\prime}, D^{\prime}\right) \in \mathcal{S}_{+}^{1}}\left(\mathbb{E}\left[-\int_{[s, T]} X_{t-} L_{t-} d D_{t}-\int_{[s, T]} X_{t} L_{t}^{\prime} d D_{t}^{\prime}\right]-\gamma\left(L, D, L^{\prime}, D^{\prime}\right)\right)
$$

and $\rho$ is cash additive up to time $s$, i.e., at all times $t \in[0, s]$.

In particular, $\rho$ is cash additive if and only if it reduces to a risk measure on $L^{\infty}\left(\Omega, \mathcal{F}_{T}, \mathbb{P}\right)$, i.e., $\rho$ is of the form

$$
\rho(X)=\sup _{Q \in \mathcal{M}(\mathbb{P})}\left(\mathbb{E}_{Q}\left[-X_{T}\right]-\tilde{\gamma}(Q)\right),
$$

where $\mathcal{M}(\mathbb{P})$ denotes the set of all probability measures on $\left(\Omega, \mathcal{F}_{T}\right)$ that are absolutely continuous with respect to $\mathbb{P}$, and $\tilde{\gamma}$ is a penalty function on $\mathcal{M}(\mathbb{P})$. 
Proof. Since $L$ and $L^{\prime}$ are local martingales, and $D$ and $D^{\prime}$ non-increasing processes with $D_{0-}=D_{0-}^{\prime}=1$, condition (6.3) is equivalent to

$$
\int_{(0, s)} L_{t-} d D_{t}=\int_{[0, s)} L_{t}^{\prime} d D_{t}^{\prime}=0 \quad \mathbb{P} \text {-a.s.. }
$$

Choose $\left(L, L^{\prime}, D, D^{\prime}\right) \in \mathcal{S}_{+}^{1}$ such that $\gamma\left(L, L^{\prime}, D, D^{\prime}\right)<\infty$, and assume that condition (6.6) does not hold. Then

$$
\mathbb{E}\left[\int_{[s, T]} L_{t-} d D_{t}+\int_{[s, T]} L_{t}^{\prime} d D_{t}^{\prime}\right]>-1,
$$

and we can find $m \in \mathbb{R}$ such that

$$
\mathbb{E}\left[\int_{[s, T]} L_{t-} d D_{t}+\int_{[s, T]} L_{t}^{\prime} d D_{t}^{\prime}\right]-\frac{\gamma\left(L, L^{\prime}, D, D^{\prime}\right)}{m}>-1 .
$$

This implies that

$$
\begin{aligned}
\rho\left(m \mathbf{1}_{[s, T]}\right) & =m \sup _{\left(L, D, L^{\prime}, D^{\prime}\right) \in \mathcal{S}_{+}^{1}}\left(\mathbb{E}\left[-\int_{[s, T]} L_{t-} d D_{t}-\int_{[s, T]} L_{t}^{\prime} d D_{t}^{\prime}\right]-\frac{\gamma\left(L, D, L^{\prime}, D^{\prime}\right)}{m}\right) \\
& >-m
\end{aligned}
$$

which contradicts the cash additivity property at time $s$. Hence (6.6) holds, and representation (6.1) reduces to (6.5). In particular, if $\rho$ is cash additive at $T$, (6.6) amounts to $a^{\mathrm{op}}=a^{\mathrm{pr}}=0$ on $[0, T)$ for all $\left(a^{\mathrm{pr}}, a^{\mathrm{op}}\right) \in \mathcal{Z}_{1}^{d}$ such that $\gamma\left(a^{\mathrm{pr}}, a^{\mathrm{op}}\right)<\infty$. Due to Remark 4.8, in this case $L+L^{\prime}$ is a uniformly integrable martingale and defines a probability measure $Q \in \mathcal{M}(\mathbb{P})$ via $\frac{d Q}{d \mathbb{P}}:=L_{T}+L_{T}^{\prime}$. It follows as in Remark 4.8

$$
\mathbb{E}\left[-\int_{0}^{T} X_{t-} L_{t-} d D_{t}-\int_{[0, T]} X_{t} L_{t}^{\prime} d D_{t}^{\prime}\right]=\mathbb{E}_{Q}\left[X_{T}\right]
$$

for any $X \in \mathcal{R}^{\infty}$, and any $\left(L, L^{\prime}, D, D^{\prime}\right) \in \mathcal{S}_{+}^{1}$ such that $\gamma\left(L, L^{\prime}, D, D^{\prime}\right)<\infty$. This proves (6.5) with

$$
\tilde{\gamma}(Q):=\gamma\left(\frac{1}{2} \frac{d Q}{d \mathbb{P}}, \frac{1}{2} \frac{d Q}{d \mathbb{P}}, 1-\delta_{\{T\}}, 1-\delta_{\{T\}}\right), \quad Q \in \mathcal{M}(\mathbb{P}),
$$

where $\delta_{\{T\}}$ denotes the Dirac measure at $T$.

\section{Risk measures and BSDEs}

This section links risk measures for processes to BSDEs. We consider here risk measures in the dynamic framework. For $0 \leq t \leq s \leq T$, we define the projection $\pi_{t, s}: \mathcal{R}^{\infty} \rightarrow \mathcal{R}^{\infty}$ as

$$
\pi_{t, s}(X)_{r}=\mathbf{1}_{[t, T]}(r) X_{r \wedge s}, \quad r \in[0, T]
$$

and we use the notation $\mathcal{R}_{t, s}^{\infty}:=\pi_{t, s}\left(\mathcal{R}^{\infty}\right)$, and $\mathcal{R}_{t}^{\infty}:=\pi_{t, T}\left(\mathcal{R}^{\infty}\right)$. Risk assessment at time $t$ takes into account the available information, and is described by a conditional convex risk measure for processes $\rho_{t}$. 
Definition 7.1. A map $\rho_{t}: \mathcal{R}_{t}^{\infty} \rightarrow L^{\infty}\left(\Omega, \mathcal{F}_{t}, \mathbb{P}\right)$ for $t \in(0, T]$ is called a conditional convex risk measure for processes if it satisfies the following properties for all $X, Y \in \mathcal{R}_{t}^{\infty}$ :

- Conditional cash invariance: for all $m \in L^{\infty}\left(\Omega, \mathcal{F}_{t}, \mathbb{P}\right)$,

$$
\rho_{t}\left(X+m \mathbf{1}_{[t, T]}\right)=\rho_{t}(X)-m
$$

- Monotonicity: $\rho_{t}(X) \geq \rho_{t}(Y)$ if $X \leq Y$;

- Conditional convexity: for all $\lambda \in L^{\infty}\left(\Omega, \mathcal{F}_{t}, \mathbb{P}\right)$ with $0 \leq \lambda \leq 1$,

$$
\rho_{t}(\lambda X+(1-\lambda) Y) \leq \lambda \rho_{t}(X)+(1-\lambda) \rho_{t}(Y)
$$

- Normalization: $\rho_{t}(0)=0$.

A sequence $\left(\rho_{t}\right)_{t \in[0, T]}$ is called a dynamic convex risk measure for processes if, for each $t$, $\rho_{t}: \mathcal{R}_{t}^{\infty} \rightarrow L^{\infty}\left(\Omega, \mathcal{F}_{t}, \mathbb{P}\right)$ is a conditional convex risk measure for processes.

For $X \in \mathcal{R}^{\infty}$ we use the notation

$$
\rho_{t}(X):=\rho_{t}\left(\pi_{t, T}(X)\right) .
$$

A dynamic convex risk measure for processes is called time consistent, if

$$
\rho_{t}(X)=\rho_{t}\left(X \mathbf{1}_{[t, s)}-\rho_{s} \mathbf{1}_{[s, T]}(X)\right)
$$

for all $X \in \mathcal{R}^{\infty}$, and all $t \in[0, T], s \in[t, T]$.

Remark 7.2. Also Definition 3.4 of cash subadditivity can be extended to the conditional case in a straightforward way. By the same argument as in Proposition 3.5 every conditional convex risk measure for processes is cash subadditive.

From now on we shell assume that the time horizon $T$ is finite, and the filtration $\left(\mathcal{F}_{t}\right)_{t \in[0, T]}$ is the augmentation of the filtration generated by a $d$-dimensional Brownian motion $\left(W_{t}\right)_{t \in[0, T]}$. In this context, it is well known that a solution to a BSDE

$$
Y_{t}=-X_{T}+\int_{t}^{T} g\left(s, Y_{s}, Z_{s}\right) d s-\int_{t}^{T} Z_{s} d W_{s}, \quad t \in[0, T],
$$

for a Lipschitz or quadratic growth driver $g=g(s, y, z)$ defines a dynamic convex risk measure for random variables, if the driver is convex in $z$ and does not depend on $y$; cf. [35, [40], [5], and the references therein. The latter requirement is due to the strong notion of cash additivity in the framework of random variables. As pointed out in [18, a solution to a BSDE (7.1) becomes cash subadditive, if the driver is monotone in $y$ and convex in $(y, z)$.

In the sequel we want to modify (17.1) in a way that it would define a dynamic convex risk measure for processes. As we have seen in Proposition 3.5, every risk measure for processes is cash subadditive; and this suggests to consider BSDEs with monotone convex drivers as in [18. However, in our framework the BSDE should depend on the whole path of the process 
$X$ rather then just on its terminal value $X_{T}$. So for a fixed $X$ in $\mathcal{R}^{\infty}$ we will consider a BSDE of the following form:

$$
Y_{t}=-X_{T}+\int_{t}^{T} g\left(s, Y_{s}+X_{s}, Z_{s}\right) d s-\int_{t}^{T} Z_{s} d W_{s}, \quad t \in[0, T] .
$$

Another example of a BSDE depending on a process is given by reflected BSDE, where the solution $Y$ of (7.1) is required to stay above an "obstacle" process $X$, cf. [17. Thus we may also add a reflection condition to the BSDE (7.2), and consider the RBSDE

$$
\begin{aligned}
& Y_{t}=-X_{T}+\int_{t}^{T} g\left(s, Y_{s}+X_{s}, Z_{s}\right) d s-\int_{t}^{T} Z_{s} d W_{s}+K_{T}-K_{t}, \quad t \in[0, T], \\
& \text { with } \\
& Y_{t} \geq-X_{t} \quad \forall t \in[0, T], \quad \text { and } \quad \int_{0}^{T}\left(Y_{s-}+X_{s-}\right) d K_{s}=0 .
\end{aligned}
$$

In the sequel we will make the following assumptions on the driver $g: \Omega \times[0, T] \times \mathbb{R} \times \mathbb{R}^{d} \rightarrow \mathbb{R}$ :

(H1)[Lipschitz] For any $(y, z) \in \mathbb{R}^{1+d}$, the stochastic process $(\omega, t) \mapsto g(\omega, t, y, z)$ is progressively measurable. In addition, there exists $C_{\text {Lip }}>0$, such that

$\left|g\left(\omega, t, y_{1}, z_{1}\right)-g\left(\omega, t, y_{2}, z_{2}\right)\right| \leq C_{\text {Lip }}\left(\left|y_{1}-y_{2}\right|+\left|z_{1}-z_{2}\right|\right) \quad \forall\left(y_{1}, y_{2}, z_{1}, z_{2}\right) \in \mathbb{R}^{2+2 d} \mathbb{P} \otimes d t$-a.e..

(H1')[Quadratic growth] For any $(y, z) \in \mathbb{R}^{1+d}$, the stochastic process $(\omega, t) \mapsto g(\omega, t, y, z)$ is progressively measurable. In addition, there exists $C>0$, such that

$$
|g(\omega, t, y, z)| \leq C\left(1+|y|+|z|^{2}\right) \quad \forall(y, z) \in \mathbb{R}^{1+d} \mathbb{P} \otimes d t \text {-a.e. }
$$

(H2) [Convexity] $g$ is convex in $(y, z)$, i.e., $\forall\left(y_{1}, y_{2}, z_{1}, z_{2}, \lambda\right) \in \mathbb{R}^{2+2 d} \times[0,1]$, $g\left(\omega, t, \lambda y_{1}+(1-\lambda) y_{2}, \lambda z_{1}+(1-\lambda) z_{2}\right) \leq \lambda g\left(\omega, t, y_{1}, z_{1}\right)+(1-\lambda) g\left(\omega, t, y_{2}, z_{2}\right) \quad \mathbb{P} \otimes d t$-a.e..

(H3) [Monotonicity] $g$ non-increasing in $y$.

(H4) [Normalization] $g(\omega, t, 0,0)=0 \quad \mathbb{P} \otimes d t$-a.s..

Before recalling existence result for the equations under interest, we point out that assumptions (H1) and (H1') from one hand, and assumptions (H2)-(H4) on the other hand are not of the same nature. Indeed, as it will be seen in the sequel, (H1) (resp. (H1')) guarantees existence and uniqueness of a (maximal) solution, whereas assumptions (H2)-(H4) ensure that the solution satisfies the basic axioms of a risk measure for processes.

Remark 7.3. In BSDEs (7.2) and (7.3) a given process $X$ shifts the driver $g$. However, for each $X \in \mathcal{R}^{\infty}$ we can define a new driver $h^{X}: \Omega \times[0, T] \times \mathbb{R} \times \mathbb{R}^{d} \rightarrow \mathbb{R}$ as

$$
h^{X}(\omega, t, y, z):=g\left(\omega, t, y+X_{t}(\omega), z\right) .
$$

By definition, $h^{X}$ directly inherits properties (H1)-(H3) (or (H1')-(H3)) from g for each $X \in \mathcal{R}^{\infty}$, and the BSDEs (7.2) and (7.3) can be written in the more conventional form in terms of the driver $h^{X}$. 
Proposition 7.4. Under assumption (H1) (resp. (H1')), there exists for each $X \in \mathcal{R}^{\infty}$ a unique triple $(Y, Z, K)$ in $\mathcal{S}^{2} \times \mathcal{H}_{d}^{2} \times \mathcal{S}_{\uparrow}^{2}$, that is a solution of the RBSDE (7.3) (resp. a unique couple $(Y, Z)$ in $\mathcal{S}^{2} \times \mathcal{H}_{d}^{2}$, that is a maximal solution of the BSDE (17.2)). Here

$$
\begin{aligned}
& \mathcal{S}^{2}:=\left\{X:=\left(X_{t}\right)_{t \in[0, T]} \mid X \text { progressively measurable, càdlàg, } \mathbb{E}\left[\sup _{t \in[0, T]}\left|X_{t}\right|^{2}\right]<\infty\right\}, \\
& \mathcal{H}_{d}^{2}:=\left\{X:=\left(X_{t}\right)_{t \in[0, T]} \mid X \text { progressively measurable, d-dim., } \mathbb{E}\left[\int_{0}^{T}\left|X_{t}\right|^{2} d t\right]<\infty\right\},
\end{aligned}
$$

and $\mathcal{S}_{\uparrow}^{2}$ denotes the subset of elements in $\mathcal{S}^{2}$ which are non-decreasing.

Proof. Using Remark 7.3, existence and uniqueness follow from classical results such as [23, 31, 36]) for the RBSDE (7.3) under (H1), and [30] for the BSDE (7.2) under (H1').

Remark 7.5. To stress the dependence on a given process $X \in \mathcal{R}^{\infty}$, we will sometimes denote the BSDEs (7.2) and (7.3) by BSDE(X), and the solution $Y$ of the BSDE(X) at time $t$ by $Y_{t}(X)$. Note that by uniqueness of the (maximal) solution on $[t, T]$, we have $Y_{t}(X)=Y_{t}\left(\pi_{t, T}(X)\right)$, which is in line with our convention $\rho_{t}(X)=\rho_{t}\left(\pi_{t, T}(X)\right)$.

For $0 \leq s \leq t \leq T$, we will also write $Y_{s, t}(X)$ to denote the solution of $B S D E(X)$ on $[0, t]$ at time s. Accordingly, $Y_{s, t}(X)=Y_{s, t}\left(\pi_{s, t}(X)\right)$, and $Y_{t}=Y_{t, T}$.

The next proposition identifies the (maximal) solution $Y=Y(X)$ of (7.2) and (7.3) as a dynamic risk measure for processes.

Proposition 7.6. Under the assumptions (H1)-(H4) (resp. (H1')-(H4)), the (maximal) solution $\left(Y_{t}\right)_{t \in[0, T]}$ of the RBSDE (7.3) (resp. of the BSDE (7.2) ) defines a time consistent dynamic convex risk measure for processes via

$$
\rho_{t}(X):=Y_{t}(X), \quad t \in[0, T], \quad X \in \mathcal{R}^{\infty} .
$$

Proof. We only deal with the reflected case here, and simply indicate the main arguments for the non-reflected quadratic growth case.

(i) To prove convexity, let $X^{1}, X^{2} \in \mathcal{R}^{\infty}$ and $\lambda \in[0,1]$; we have to show that

$$
Y\left(\lambda X^{1}+(1-\lambda) X^{2}\right) \leq \lambda Y\left(X^{1}\right)+(1-\lambda) Y\left(X^{2}\right) .
$$

To this end we denote by $\left(Y^{i}, Z^{i}, K^{i}\right)$ the solutions of the BSDE (17.3) for $X=X^{i}(i=1,2)$, and set $\tilde{X}:=\lambda X^{1}+(1-\lambda) X^{2}, \tilde{Y}:=\lambda Y\left(X^{1}\right)+(1-\lambda) Y\left(X^{2}\right), \tilde{Z}:=\lambda Z^{1}+(1-\lambda) Z^{2}$, and $\tilde{K}:=\lambda K^{1}+(1-\lambda) K^{2}$. Convexity of $g$ in $(y, z)$ implies

$$
\lambda g\left(r, Y_{r}^{1}+X_{r}^{1}, Z_{r}^{1}\right)+(1-\lambda) g\left(r, Y_{r}^{2}+X_{r}^{2}, Z_{r}^{2}\right) \geq g\left(r, \tilde{Y}_{r}+\tilde{X}_{r}, \tilde{Z}_{r}\right), \quad \mathbb{P} \text {-a.s.. }
$$

Thus we have for any $0 \leq t_{1} \leq t_{2} \leq T$

$$
\begin{aligned}
\tilde{Y}_{t_{1}} & =\tilde{Y}_{t_{2}}+\int_{t_{1}}^{t_{2}}\left(\lambda g\left(r, Y_{r}^{1}+X_{r}^{1}, Z_{r}^{1}\right)+(1-\lambda) g\left(r, Y_{r}^{2}+X_{r}^{2}, Z_{r}^{2}\right)\right) d r-\int_{t_{1}}^{t_{2}} \tilde{Z}_{r} d W_{r}+\int_{t_{1}}^{t_{2}} d \tilde{K}_{r} \\
& \geq \tilde{Y}_{t_{2}}+\int_{t_{1}}^{t_{2}} g\left(r, \tilde{Y}_{r}+\tilde{X}_{r}, \tilde{Z}_{r}\right) d r-\int_{t_{1}}^{t_{2}} \tilde{Z}_{r} d W_{r} .
\end{aligned}
$$


Hence $\tilde{Y}$ is a supersolution of the classical BSDE with driver $g$ and terminal condition $\tilde{X}_{T}$, and $\tilde{Y} \geq \tilde{X}$. As it is proved in [36, Theorem 2.1], $Y\left(\lambda X^{1}+(1-\lambda) X^{2}\right)$ is the smallest supersolution of the (classical) BSDE with driver $g$ and terminal condition $\tilde{X}_{T}$ which dominates $\tilde{X}$. Thus

$$
\tilde{Y}_{t} \geq Y_{t}\left(\lambda X^{1}+(1-\lambda) X^{2}\right) \quad \forall t \in[0, T] \quad \mathbb{P} \text {-a.s.. }
$$

In the non-reflected case, comparison theorem for maximal solutions of BSDEs (c.f., e.g., [18, Theorem 7.1]) provides the result.

(ii) To prove (inverse) monotonicity, note that for any $X^{1}, X^{2} \in \mathcal{R}^{\infty}$ such that $X^{1} \leq X^{2}$ we have $Y_{T}\left(X^{1}\right) \geq Y_{T}\left(X^{2}\right)$. Moreover, since $g$ is non-increasing in $y$, we have $h^{X^{1}}(t, y, z) \geq$ $h^{X^{2}}(t, y, z)$ for all $(t, y, z)$. Thus monotonicity follows form the classical comparison principle for (R)BSDEs, cf., e.g., [18, Theorem 7.1] and [23, Theorem 1.5].

(iii) We prove cash additivity at time $t$, i.e.,

$$
Y_{t}\left(X+m \mathbf{1}_{[t, T]}\right)=Y_{t}(X)-m \quad \forall m \in L^{\infty}\left(\Omega, \mathcal{F}_{t}, \mathbb{P}\right) .
$$

Let $(\tilde{Y}, \tilde{Z}, \tilde{K})$ denote the solution of $\operatorname{RBSDE}\left(X+m \mathbf{1}_{[t, T]}\right)$. By definition, it holds that

$$
\tilde{Y}_{s}+m=-X_{T}+\int_{s}^{T} g\left(r, \tilde{Y}_{r}+X_{r}+m, \tilde{Z}_{r}\right) d r-\int_{s}^{T} \tilde{Z}_{r} d W_{r}+\int_{s}^{T} d \tilde{K}_{s}, \quad s \in[t, T] .
$$

Thus $(\tilde{Y}+m, \tilde{Z}, \tilde{K})$ is the solution of (7.3) on $[t, T]$, and by uniqueness $\tilde{Y}_{t}+m=Y_{t}(X)$. (iv) Due to the requirement $g(t, 0,0)=0 \mathbb{P} \otimes d t$-a.s., $(0,0,0)$ is the unique solution to the $\operatorname{BSDE}(0)$; this proves normalization.

(v) We prove time consistency:

$$
Y_{t}\left(X \mathbf{1}_{[t, s)}-Y_{s}(X) \mathbf{1}_{[s, T]}(X)\right)=Y_{t}(X) \quad \forall t \in[0, T], s \in[t, T] .
$$

To this end, we first show that for $s \in[t, T]$

$$
Y_{t, T}(X)=Y_{t, s}\left(X \mathbf{1}_{[t, s)}-Y_{s, T}(X) \mathbf{1}_{[s]}\right)
$$

Indeed, if $(Y, Z, K)$ denotes the solution of $\operatorname{RBSDE}(X)$, we have

$$
\begin{aligned}
Y_{t, T}(X)=-X_{T} & +\int_{s}^{T} g\left(r, Y_{r}+X_{r}, Z_{r}\right) d r-\int_{s}^{T} Z_{r} d W_{r}+\int_{s}^{T} d K_{r} \\
& +\int_{t}^{s} g\left(r, Y_{r}+X_{r}, Z_{r}\right) d r-\int_{t}^{s} Z_{r} d W_{r}+\int_{t}^{s} d K_{r} \\
= & Y_{s, T}(X)+\int_{t}^{s} g\left(r, Y_{r}+X_{r}, Z_{r}\right) d r-\int_{t}^{s} Z_{r} d W_{r}+\int_{t}^{s} d K_{r} \\
= & Y_{t, s}\left(X \mathbf{1}_{[t, s)}-Y_{s, T}(X) \mathbf{1}_{[s]}\right)
\end{aligned}
$$

due to uniqueness of the solution. Now let $(\tilde{Y}, \tilde{Z}, \tilde{K})$ denote the solution of the $\operatorname{RBSDE}\left(X \mathbf{1}_{[0, s)}-\right.$ $\left.Y_{s, T}(X) \mathbf{1}_{[s, T]}\right)$. Then we have

$$
\tilde{Y}_{t}=Y_{s, T}(X)+\int_{t}^{s} g\left(r, \tilde{Y}_{r}+X_{r}, \tilde{Z}_{r}\right) d r-\int_{t}^{s} \tilde{Z}_{r} d W_{r}+\int_{t}^{s} d \tilde{K}_{r}
$$




$$
-Y_{s, T}(X)+Y_{s, T}(X)+\int_{s}^{T} g\left(r, \tilde{Y}_{r}-Y_{s, T}(X), \tilde{Z}_{r}\right) d r-\int_{s}^{T} \tilde{Z}_{r} d W_{r}+\int_{s}^{T} d \tilde{K}_{r} .
$$

Note further that (7.5) equals to $Y_{t, T}(X)$ by (7.4), and (7.6) is 0 , since

$$
\begin{aligned}
Y_{s, T}(X)+\int_{s}^{T} g\left(r, \tilde{Y}_{r}-Y_{s, T}(X), \tilde{Z}_{r}\right) d r-\int_{s}^{T} \tilde{Z}_{r} d W_{r}+\int_{s}^{T} d \tilde{K}_{r} & =Y_{s}\left(-Y_{s, T}(X) \mathbf{1}_{[s, T]}\right) \\
& =Y_{s, T}(X)
\end{aligned}
$$

due to cash invariance and normalization as proved in (iii) and (iv).

In the following we will provide dual representations for the risk measures associated to the BSDEs (7.2) and (7.3). To this end we define the Legendre-Fenchel conjugate $g^{*}$ : $\Omega \times[0, T] \times \mathbb{R} \times \mathbb{R}^{d} \rightarrow \mathbb{R} \cup\{\infty\}$ of the convex generator $g$ as in [18]:

$$
\begin{aligned}
g^{*}(\omega, t, \beta, \mu) & =\sup _{(y, z) \in \mathbb{R}^{\prime} \mathbb{R}^{d}}\{-\beta y-\mu \cdot z-g(\omega, t, y, z)\} \\
& =\sup _{(y, z) \in \mathbb{Q} \times \mathbb{Q}^{d}}\{-\beta y-\mu \cdot z-g(\omega, t, y, z)\} .
\end{aligned}
$$

Moreover, we introduce the sets

$$
\mathcal{R}:=\left\{\beta=\left(\beta_{t}\right)_{t \in[0, T]} \mid \beta \text { progressively measurable, } 0 \leq \beta \leq C \mathbb{P} \otimes d t \text {-a.s. }\right\},
$$

and

$$
\operatorname{BMO}(\mathbb{P}):=\left\{\mu=\left(\mu_{t}\right)_{t \in[0, T]} \mid \mu \in \mathcal{H}_{d}^{2}, \exists B: \sup _{\tau \text { stopping time }} \mathbb{E}\left[\int_{\tau}^{T}\left|\mu_{s}\right|^{2} d s \mid \mathcal{F}_{\tau}\right] \leq B \mathbb{P} \text {-a.s. }\right\} .
$$

Lemma 7.7. Assume that g satisfies conditions (H1)-(H4) (resp. (H1')-(H4)), and let $(Y, Z, K)$ (resp. $(Y, Z))$ be a solution to the BSDE (7.3) (resp. to (7.2) for a process $X \in$ $\mathcal{R}^{\infty}$. Then

$$
g\left(t, Y_{t}+X_{t}, Z_{t}\right)=\max _{(\beta, \mu) \in \mathcal{R} \times \mathrm{BMO}(\mathbb{P})}\left\{-\beta_{t}\left(Y_{t}+X_{t}\right)-\mu_{t} \cdot Z_{t}-g^{*}\left(t, \beta_{t}, \mu_{t}\right)\right\} \quad \mathbb{P} \otimes d t \text {-a.s. }
$$

where the maximum is attained by some $(\bar{\beta}, \bar{\mu}) \in \mathcal{R} \times \mathrm{BMO}(\mathbb{P})$.

Proof. Note first that (H1) together with (H4) implies (H1'), so it is sufficient to argue for $g$ satisfying quadratic growth condition (H1'). By definition of $g^{*}$, we have " $\geq$ " in (7.7), and standard convex duality and measurable selection results (cf. [5, Lemma 7.5]) imply

$$
g\left(t, Y_{t}+X_{t}, Z_{t}\right)=-\bar{\beta}_{t}\left(Y_{t}+X_{t}\right)-\bar{\mu}_{t} \cdot Z_{t}-g^{*}\left(t, \bar{\beta}_{t}, \bar{\mu}_{t}\right) \quad \mathbb{P} \otimes d t \text {-a.s. }
$$

for some progressively measurable processes $\bar{\beta}$ and $\bar{\mu}$. We have to show that $0 \leq \bar{\beta} \leq C$ and $\bar{\mu} \in \mathrm{BMO}(\mathbb{P})$. The first estimate follows from [18, Lemma 7.4], since $g^{*}(t, \beta, \mu)=\infty$ for $\beta \notin[0, C]$. Moreover, the same argument as in [18, Lemma 7.4] implies that there exists $B>0$ such that

$$
\left|\bar{\mu}_{t}^{2}\right| \leq B\left(1+\left|Y_{t}\right|+\left|X_{t}\right|+\left|Z_{t}\right|^{2}\right) \quad \mathbb{P} \otimes d t \text {-a.s. }
$$

As proved in the appendix, $Y$ is bounded, and $Z \in \operatorname{BMO}(\mathbb{P})$ for each $X \in \mathcal{R}^{\infty}$ both in (7.3) and in (7.2). This proves that $\bar{\mu} \in \mathrm{BMO}(\mathbb{P})$. 
By classical results of Kazamaki [29, Section 3.3], cf. also [5, Theorem 7.2], every $\mu \in$ $\mathrm{BMO}(\mathbb{P})$ defines a probability measure $Q^{\mu} \approx \mathbb{P}$ on $\mathcal{F}_{T}$ via the density process

$$
\Gamma_{t}^{\mu}=\exp \left(\int_{0}^{t} \mu_{s} d W_{s}-\frac{1}{2} \int_{0}^{t}\left|\mu_{s}\right|^{2} d s\right), \quad t \in[0, T] .
$$

Moreover, $W^{\mu}:=W-\int_{0}^{\cdot} \mu_{s} d s$ is a $Q^{\mu}$-Brownian motion, and $\int_{0}^{\cdot} Z_{s} d W_{s}^{\mu}$ is a $\operatorname{BMO}\left(Q^{\mu}\right)$ martingale for any $Z \in \mathrm{BMO}(\mathbb{P})$.

Probability measures $Q^{\mu}$ will describe models appearing in the dual representations of the risk measures associated to BSDEs (17.3) and (7.2). We also define for each $t \in[0, T]$ a family of discounting process

$$
\begin{gathered}
\mathcal{D}_{t}:=\left\{\left(D_{t, s}\right)_{s \in[t, T]} \mid\left(D_{t, s}\right)\right. \text { adapted, non-increasing, right-continuous, } \\
\left.D_{t, t-}:=1, D_{t, T}=0 \mathbb{P} \text {-a.s. }\right\} .
\end{gathered}
$$

Every $D \in \mathcal{D}_{0}$ and a density process $\Gamma^{\mu}$ as above define a normalized optional measure $\nu$ as in Corollary 4.3 and (4.24) via

$$
\mathbb{E}_{\nu}[X]=\mathbb{E}\left[-\int_{[0, T]} X_{s} \Gamma_{s}^{\mu} d D_{0, s}\right]=\mathbb{E}_{Q^{\mu}}\left[-\int_{[0, T]} X_{s} d D_{0, s}\right], \quad X \in \mathcal{R}^{\infty} .
$$

If we define $\overline{\mathcal{F}}_{t}:=\sigma\left(\pi_{0, t}(X) \mid X \in \mathcal{R}^{\infty}\right)$, and $\left(D_{t, s}\right) \in \mathcal{D}_{t}$ via $D_{t, s}:=\frac{D_{0, s}}{D_{0, t-}}, s \in[t, T]$, $\overline{\mathcal{F}}_{t}$-conditional expectation with respect to $\nu$ can be written as

$$
\mathbb{E}_{\nu}\left[X \mid \overline{\mathcal{F}}_{t}\right]=X \mathbf{1}_{[0, t)}+\mathbb{E}_{Q^{\mu}}\left[-\int_{[t, T]} X_{s} d D_{t, s} \mid \mathcal{F}_{t}\right] \mathbf{1}_{[t, T]}, \quad X \in \mathcal{R}^{\infty} .
$$

For $X \in \mathcal{R}_{t}^{\infty}$, this conditional expectation reduces to $\mathbb{E}_{Q^{\mu}}\left[-\int_{[t, T]} X_{s} d D_{t, s} \mid \mathcal{F}_{t}\right]$, and it will appear in the conditional dual representation of the dynamic risk measures induced by BSDEs (7.2) and (7.3). To be more precise, we will show that the risk measures induced by BSDEs (7.2) and (7.3) are of the form

$$
\rho_{t}(X)=\operatorname{ess~sup}_{(\mu, D) \in \operatorname{BMO}(\mathbb{P}) \times \mathcal{D}_{t}}\left(E_{Q^{\mu}}\left[\int_{[t, T]} X_{s} d D_{t, s} \mid \mathcal{F}_{t}\right]-\gamma_{t}(\mu, D)\right), \quad X \in \mathcal{R}^{\infty},
$$

where $\gamma_{t}(\mu, D)$ is a penalty function on $\operatorname{BMO}(\mathbb{P}) \times \mathcal{D}_{t}$, and $t \in[0, T]$. This representation can be seen as a conditional version of (6.2), where the penalty function is concentrated on the local martingales of the form $\Gamma^{\mu}$, i.e., on probability measures $Q^{\mu}$, that are equivalent to the Wiener measure $\mathbb{P}$.

In order to prove (7.8), let $(Y, Z, K)$ be the (maximal) solution of the $\operatorname{BSDE}(X)$, fix $\mu \in \operatorname{BMO}(\mathbb{P})$ and $D \in \mathcal{D}_{t}$. Applying integration by parts, taking conditional expectation with respect to $Q^{\mu}$ on both sides, and using that $\int_{0}^{\cdot} Z_{s} d W_{s}^{\mu}$ is a $\operatorname{BMO}\left(Q^{\mu}\right)$-martingale, we obtain

$$
Y_{t}=Y_{t} D_{t, t-}=-Y_{t} \Delta D_{t, t}+Y_{t} D_{t, t}
$$




$$
\begin{aligned}
= & \mathbb{E}_{Q^{\mu}}\left[-Y_{t} \Delta D_{t, t}+D_{t, T} Y_{T}-\int_{t}^{T} Y_{s} d D_{t, s}-\int_{t}^{T} D_{t, s-} d Y_{s} \mid \mathcal{F}_{t}\right] \\
= & \mathbb{E}_{Q^{\mu}}\left[\int_{[t, T]} X_{s} d D_{t, s} \mid \mathcal{F}_{t}\right] \\
& +\mathbb{E}_{Q^{\mu}}\left[\int_{t}^{T} D_{t, s-}\left(g\left(s, Y_{s}+X_{s}, Z_{s}\right)+\mu_{s} \cdot Z_{s}\right) d s \mid \mathcal{F}_{t}\right] \\
& +\mathbb{E}_{Q^{\mu}}\left[-\int_{[t, T]}\left(Y_{s}+X_{s}\right) d D_{t, s}+\int_{t}^{T} D_{t, s-} d K_{s} \mid \mathcal{F}_{t}\right],
\end{aligned}
$$

where the $d K$ term in (7.11) disappears for the non-reflected BSDE (7.2). These computations lead to the following examples.

Example 7.8. We consider the BSDE (7.2)

$$
Y_{t}=-X_{T}+\int_{t}^{T} g\left(s, Y_{s}+X_{s}, Z_{s}\right) d s-\int_{t}^{T} Z_{s} d W_{s} \quad t \in[0, T]
$$

where the driver $g$ satisfies assumptions (H1')-(H4). This is the same framework as in [18, Section 7], but in our case the BSDE depends on the whole path of the process $X \in \mathcal{R}^{\infty}$. The results from [18] follow from our considerations if applied to processes $X:=X_{T} \mathbf{1}_{[T]}$ for $X_{T} \in L^{\infty}\left(\Omega, \mathcal{F}_{T}, \mathbb{P}\right)$.

For $\beta \in \mathcal{R}$ and $t \in[0, T]$, we introduce the discounting factors

$$
D_{t, s}:=e^{-\int_{t}^{s} \beta_{u} d u}, \quad s \in[t, T), \quad \text { and } \quad D_{t, T}=0 .
$$

Note that $\left(D_{t, s}\right) \in \mathcal{D}_{t}$ for all $t$.

Theorem 7.9. The BSDE (7.2) induces under assumptions(H1')-(H4) a dynamic convex risk measure for processes $\left(\rho_{t}\right)_{t \in[0, T]}$ with the robust representation

$$
\begin{aligned}
\rho_{t}(X)=Y_{t}=\operatorname{essup}_{(\mu, \beta) \in \operatorname{BMO}(\mathbb{P}) \times \mathcal{R}} & \left(\mathbb{E}_{Q^{\mu}}\left[e^{-\int_{t}^{T} \beta_{u} d u}\left(-X_{T}\right)-\int_{t}^{T} \beta_{s} X_{s} e^{-\int_{t}^{s} \beta_{u} d u} d s \mid \mathcal{F}_{t}\right]\right. \\
& \left.-\mathbb{E}_{Q^{\mu}}\left[\int_{t}^{T} e^{-\int_{t}^{s} \beta_{u} d u} g^{*}\left(s, \beta_{s}, \mu_{s}\right) d s \mid \mathcal{F}_{t}\right]\right),
\end{aligned}
$$

where the essential supremum is attained for each $X \in \mathcal{R}^{\infty}$ by some $(\bar{\mu}, \bar{\beta}) \in \operatorname{BMO}(\mathbb{P}) \times \mathcal{R}$. Proof. Applying (7.9), (7.10), and (7.11) with $\left(D_{t, s}\right)$ defined in (7.12), we obtain

$$
\begin{aligned}
Y_{t}= & \mathbb{E}_{Q^{\mu}}\left[e^{-\int_{t}^{T} \beta_{u} d u}\left(-X_{T}\right)-\int_{t}^{T} \beta_{s} X_{s} e^{-\int_{t}^{s} \beta_{u} d u} d s \mid \mathcal{F}_{t}\right] \\
& +\mathbb{E}_{Q^{\mu}}\left[\int_{t}^{T} e^{-\int_{t}^{s} \beta_{u} d u}\left(g\left(s, Y_{s}+X_{s}, Z_{s}\right)+\beta_{s}\left(Y_{s}+X_{s}\right)+\mu_{s} \cdot Z_{s}\right) d s \mid \mathcal{F}_{t}\right] .
\end{aligned}
$$

By Lemma 7.7 (7.14) $\geq(7.13)$ for all $(\mu, \beta) \in \mathrm{BMO}(\mathbb{P}) \times \mathcal{R}$, with equality attained at some optimal $(\bar{\mu}, \bar{\beta})$. 
Remarks 7.10. 1. Theorem 7.9 follows also directly from [18, Theorem 7.5], applied to the driver $h^{X}(t, y, z)=g\left(t, y+X_{t}, z\right)$ defined in Remark 7.3. Indeed, we have for all $\omega, t, \beta$, and $\mu$

$$
\left(h^{X}\right)^{*}(\omega, t, \beta, \mu)=\beta X_{t}(\omega)+g^{*}(\omega, t, \beta, \mu) .
$$

2. Note that $\rho_{t}$ in Theorem 7.9 is of the form (7.8), with penalty function

$$
\gamma_{t}\left(Q^{\mu}, D\right)=\gamma_{t}(\mu, D)=\gamma_{t}(\mu, \beta)=\mathbb{E}_{Q^{\mu}}\left[\int_{t}^{T} e^{-\int_{t}^{s} \beta_{u} d u} g^{*}\left(s, \beta_{s}, \mu_{s}\right) d s \mid \mathcal{F}_{t}\right] .
$$

This penalty function is concentrated on discounting measures $d D$, that are absolutely continuous with respect to the Lebesgue measure $\lambda$. This is due to the fact that the process $X$ appears only in the driver of (7.2), i.e., in the $\lambda$-absolutely continuous part of the BSDE.

Example 7.11. In this example we consider the BSDE (7.3)

$$
\begin{aligned}
& Y_{t}=-X_{T}+\int_{t}^{T} g\left(s, Y_{s}+X_{s}, Z_{s}\right) d s-\int_{t}^{T} Z_{s} d W_{s}+K_{T}-K_{t}, \quad t \in[0, T], \\
& Y_{t} \geq-X_{t} \quad \forall t \in[0, T], \quad \text { and } \quad \int_{0}^{T}\left(Y_{s-}+X_{s-}\right) d K_{s}=0 .
\end{aligned}
$$

For each $t \in[0, T]$, we define the set of stopping times

$$
\Theta_{t}:=\{\tau \mid \tau \text { is a stopping time, } t \leq \tau \leq T \text { P-a.s. }\},
$$

and for $\tau \in \Theta_{t}$ and $\beta \in \mathcal{R}$ the discounting factors $D \in \mathcal{D}_{t}$ via

$$
D_{t, t-}:=1, \quad D_{t, s}:=e^{-\int_{t}^{s} \beta_{s} d s} \mathbf{1}_{\{\tau>s\}}, \quad s \in[t, T] .
$$

Theorem 7.12. The BSDE (7.3) induces under assumptions (H1)-(H4) a dynamic convex risk measure for processes $\left(\rho_{t}\right)_{t \in[0, T]}$ with the robust representation

$$
\begin{aligned}
\rho_{t}(X)=Y_{t}=\operatorname{ess}_{(\mu, \beta, \tau) \in \operatorname{BMO}(\mathbb{P}) \times \mathcal{R} \times \Theta_{t}} & \left(\mathbb{E}_{Q^{\mu}}\left[e^{-\int_{t}^{\tau} \beta_{u} d u}\left(-X_{\tau}\right)-\int_{t}^{\tau} \beta_{s} X_{s} e^{-\int_{t}^{s} \beta_{u} d u} d s \mid \mathcal{F}_{t}\right]\right. \\
& \left.-\mathbb{E}_{Q^{\mu}}\left[\int_{t}^{\tau} e^{-\int_{t}^{s} \beta_{u} d u} g^{*}\left(s, \beta_{s}, \mu_{s}\right) d s \mid \mathcal{F}_{t}\right]\right)
\end{aligned}
$$

for all $X \in \mathcal{R}^{\infty}$.

Proof. Applying (7.9), (7.10), and (7.11) with $\left(D_{t, s}\right)$ defined in (7.15), and using $d D_{t, s}=$ $-\mathbf{1}_{\{s \leq \tau\}} \beta_{s} e^{-\int_{t}^{s} \beta_{u} d u} d s-e^{-\int_{t}^{\tau} \beta_{u} d u} \delta_{\{\tau\}}(d s)$, and $D_{t, s-}=e^{-\int_{t}^{s} \beta_{u} d u} \mathbf{1}_{\{\tau \geq s\}}$, we obtain

$$
\begin{aligned}
Y_{t}= & \mathbb{E}_{Q^{\mu}}\left[e^{-\int_{t}^{\tau} \beta_{u} d u}\left(-X_{\tau}\right)-\int_{t}^{\tau} \beta_{s} X_{s} e^{-\int_{t}^{s} \beta_{u} d u} d s \mid \mathcal{F}_{t}\right] \\
& +\mathbb{E}_{Q^{\mu}}\left[\int_{t}^{\tau} e^{-\int_{t}^{s} \beta_{u} d u}\left(g\left(s, Y_{s}+X_{s}, Z_{s}\right)+\beta_{s}\left(Y_{s}+X_{s}\right)+\mu_{s} \cdot Z_{s}\right) d s \mid \mathcal{F}_{t}\right]
\end{aligned}
$$




$$
+\mathbb{E}_{Q^{\mu}}\left[e^{-\int_{t}^{\tau} \beta_{u} d u}\left(Y_{\tau}+X_{\tau}\right)+\int_{t}^{\tau} e^{-\int_{t}^{s} \beta_{u} d u} d K s \mid \mathcal{F}_{t}\right] .
$$

By Lemma 7.7, (7.17) $\geq(7.16)$ for all $(\mu, \beta, \tau)$, with equality attained independently of $\tau$ at some $(\bar{\mu}, \bar{\beta}) \in \operatorname{BMO}(\mathbb{P}) \times \mathcal{R}$. Moreover, since $Y_{t}+X_{t} \geq 0$ for all $t$, and $K$ is non-decreasing, $(7.18) \geq 0$ for all $\tau \in \Theta_{t}$; this proves " $\geq$ " in the representation. On the other hand, for any $\varepsilon>0$ we can define the stopping time

$$
\tau^{\varepsilon}:=\inf \left\{s \geq t \mid Y_{s} \leq-X_{s}+\varepsilon\right\} \in \Theta_{t} .
$$

It follows as in the proof of [31, Proposition 3.1] that $K_{\tau^{\varepsilon}}-K_{t}=0$, and hence

$$
\mathbb{E}_{Q^{\bar{\mu}}}\left[e^{-\int_{t}^{\tau^{\varepsilon}} \bar{\beta}_{u} d u}\left(Y_{\tau^{\varepsilon}}+X_{\tau^{\varepsilon}}\right)+\int_{t}^{\tau^{\varepsilon}} e^{-\int_{t}^{s} \bar{\beta}_{u} d u} d K s \mid \mathcal{F}_{t}\right] \leq \varepsilon .
$$

This shows that the right-hand-side of the representation (7.16) is larger or equal than $Y_{t}-\varepsilon$ for any $\varepsilon>0$, and proves the equality.

Example 7.13. If the generator $g$ in the previous example does not depend on $y$, the BSDE (7.3) takes the form

$$
\begin{aligned}
& Y_{t}=-X_{T}+\int_{t}^{T} g\left(s, Z_{s}\right) d s-\int_{t}^{T} Z_{s} d W_{s}+K_{T}-K_{t}, \quad t \in[0, T], \\
& Y_{t} \geq-X_{t} \quad \forall t \in[0, T], \quad \text { and } \quad \int_{0}^{T}\left(Y_{s-}+X_{s-}\right) d K_{s}=0 .
\end{aligned}
$$

In this case the conjugate $g^{*}(t, \beta, \mu)=\infty$ if $\beta \not \equiv 0$, and thus the penalty function in (17.16) is concentrated on the discounting factors $D \in \mathcal{D}_{t}$ such that $D_{t, t-}=1$, and $D_{t, s}=\mathbf{1}_{\{\tau>s\}}$ for $s \in[t, T]$ and $\tau \in \Theta_{t}$. We write $g^{*}(t, \mu):=g^{*}(t, 0, \mu)$; then Theorem 7.12 takes the following form.

Corollary 7.14. The BSDE (7.19) induces under assumptions (H1)-(H4) a dynamic convex risk measure for processes $\left(\rho_{t}\right)_{t \in[0, T]}$ with the robust representation

$$
\rho_{t}(X)=Y_{t}=\operatorname{ess~sup}_{(\mu, \tau) \in \operatorname{BMO}(\mathbb{P}) \times \Theta_{t}}\left(\mathbb{E}_{Q^{\mu}}\left[-X_{\tau} \mid \mathcal{F}_{t}\right]-\mathbb{E}_{Q^{\mu}}\left[\int_{t}^{\tau} g^{*}\left(s, \mu_{s}\right) d s \mid \mathcal{F}_{t}\right]\right)
$$

for all $X \in \mathcal{R}^{\infty}$.

This example was studied in [32, 6, 38] in the context of optimal stopping of risk measures for random variables. In our framework it appears naturally as an example of a risk measure for processes.

Example 7.15. In order to identify a BSDE as a risk measure for processes, it seems to be crucial that the driver $g$, as well as the reflection term $K$ depend on the sum $X+Y$. For instance, it was shown in [17, Section 7] for the classical RBSDE

$$
Y_{t}=-X_{T}+\int_{t}^{T} g\left(s, Y_{s}, Z_{s}\right) d s-\int_{t}^{T} Z_{s} d W_{s}+K_{T}-K_{t}, \quad t \in[0, T],
$$




$$
Y_{t} \geq-X_{t} \quad \forall t \in[0, T], \quad \text { and } \quad \int_{0}^{T}\left(Y_{s}+X_{s}\right) d K_{s}=0
$$

under the assumptions that $X$ is continuous and $g$ satisfies (H1)-(H3), that $Y$ has the dual representation

$$
Y_{t}(X)=\operatorname{ess~sup}_{(\mu, \beta, \tau) \in \operatorname{BMO}(\mathbb{P}) \times \mathcal{R} \times \Theta_{t}}\left(\mathbb{E}_{Q^{\mu}}\left[e^{-\int_{t}^{\tau} \beta_{u} d u}\left(-X_{\tau}\right)-\int_{t}^{\tau} e^{-\int_{t}^{s} \beta_{u} d u} g^{*}\left(s, \beta_{s}, \mu_{s}\right) d s \mid \mathcal{F}_{t}\right]\right) .
$$

If $g$ (resp. $g^{*}$ ) does not depend on $Y+X$, and the right-hand-side of (7.20) does not take the form as in Theorem 7.12, $Y$ does not define a conditional risk measure for processes in the sense of Definition 7.1, It does not satisfy the axiom of cash additivity.

In general, using Lebesgue decomposition, we can write every measure $d D$ induced by a discounting process $D \in \mathcal{D}_{0}$ as a sum $d D^{\ll}+d D^{\perp}$, where $d D^{\ll}$ denotes the absolutely continuous, and $d D^{\perp}$ the singular part of $d D$ with respect to the Lebesgue measure $\lambda$. For instance, for $D$ defined in (7.15) we have

$$
d D_{t, s}=\underbrace{-\mathbf{1}_{\{s \leq \tau\}} \beta_{s} e^{-\int_{t}^{s} \beta_{u} d u} d s}_{d D^{\ll}}-\underbrace{e^{-\int_{t}^{\tau} \beta_{u} d u} \delta_{\{\tau\}}(d s)}_{d D^{\perp}} .
$$

As we have noted in Remark 7.10, only absolutely continuous discounting factors $d D^{\ll}$ appear in the robust representation of the risk measure, if there is no reflection, and only the driver of the BSDE depends on the sum $Y+X$. On the other hand, as seen in Example 7.13. if there is reflection, and the driver does not depend on $Y+X$, absolutely continuous parts $d D^{\ll}$ disappear, and only singular parts $d D^{\perp}$ contribute to the robust representation.

The study of general relation between BSDEs of type (7.3) and risk measures of the form (17.8) is subject of future research. Examples presented in this paper suggest that appearance of absolutely continuous discounting factors corresponds to the dependence of the driver $g$ on the sum $Y+X$, whereas appearance of the singular discounting terms is induced by the reflection term $K$ depending on $Y+X$. Also more general reflection terms, induced by more complex penalty function on $d D^{\perp}$, can be thought about.

\section{Appendix}

We provide here estimates for the BSDEs (7.2) and (7.3), that are used in the proof of Lemma 7.7. The results for quadratic BSDE (7.2) follow basically from [5, 18]; the results for the reflected BSDE (17.3) might be known, but since we did not find them explicitly written in the literature, we give the proofs here. Throughout this section we consider a BSDE (7.2) under assumptions (H1)-(H4), and RBSDE (7.3) under assumptions (H1')-(H4).

Proposition 7.16. Let $(Y, Z, K)$ (resp. $(Y, Z))$ be the solution of (7.3) (resp. the maximal solution of (7.2) for $X \in \mathcal{R}^{\infty}$. Then $Y$ is bounded, and $Z \in \operatorname{BMO}(\mathbb{P})$.

Proof. To see that $Y$ is bounded, we use monotonicity, cash additivity, and normalization as proved in Proposition 7.6. Let $\|X\|_{\mathcal{R}^{\infty}}=: B$, then

$$
Y_{t}(X) \leq Y_{t}\left(0-B \mathbf{1}_{[t, T]}\right)=B \quad \mathbb{P} \text {-a.s. for all } t \in[0, T]
$$


and the converse inequality follows in the same manner.

The proof that $Z \in \mathrm{BMO}(\mathbb{P})$ in the non-reflected quadratic case follows as in [5. Proposition 7.3], using that $Y$ and $X$ are bounded. In the reflected case we use classical estimates, as for example in [37, where such technique is used in the context of second order BSDEs.

Itô's formula implies for any $\tau \in \Theta_{0}$ and any $\alpha>0$ that

$$
\begin{aligned}
e^{-\alpha Y_{\tau}} & =e^{-\alpha Y_{\tau}}-\alpha \int_{\tau}^{T} e^{-\alpha Y_{s}} g\left(s, Y_{s}+X_{s}, Z_{s}\right) d s+\alpha \int_{\tau}^{T} e^{-\alpha Y_{s}} Z_{s} d W_{s}-\frac{\alpha^{2}}{2} \int_{\tau}^{T} e^{-\alpha Y_{s}}\left|Z_{s}\right|^{2} d s \\
& -\alpha \int_{\tau}^{T} e^{-\alpha Y_{s-}} d K_{s}-\sum_{\tau<s \leq T}\left[e^{-\alpha Y_{s}}-e^{-\alpha Y_{s-}}+\alpha e^{-\alpha Y_{s-}} \Delta_{s} Y\right] .
\end{aligned}
$$

Since $K$ is non-decreasing, and thus $\Delta_{s} Y=\Delta_{s} K \geq 0$, and since the mapping $x \mapsto e^{-x}-1+x$ is non-negative on $\mathbb{R}_{+}$, the last two terms are non-positive. Hence (7.21) rewrites as:

$$
\frac{\alpha^{2}}{2} \int_{\tau}^{T} e^{-\alpha Y_{s}}\left|Z_{s}\right|^{2} d s+e^{-\alpha Y_{\tau}} \leq e^{-\alpha Y_{T}}-\alpha \int_{\tau}^{T} e^{-\alpha Y_{s}} g\left(s, Y_{s}+X_{s}, Z_{s}\right) d s+\alpha \int_{\tau}^{T} e^{-\alpha Y_{s}} Z_{s} d W_{s} .
$$

This implies, since $g$ has Lipschitz growth, and $X$ and $Y$ are bounded, that

$$
\frac{\alpha^{2}}{2} \int_{\tau}^{T} e^{-\alpha Y_{s}}\left|Z_{s}\right|^{2} d s \leq e^{-\alpha Y_{T}}+C \alpha \int_{\tau}^{T} e^{-\alpha Y_{s}}\left(1+\left|Z_{s}\right|^{2}\right) d s+\alpha \int_{\tau}^{T} e^{-\alpha Y_{s}} Z_{s} d W_{s},
$$

where we have used that $|x| \leq 1+|x|^{2}$. ( $C$ in this proof denotes a generic constant, which can differ from line to line.) Using again the fact that $Y$ is bounded, we get that there exists a constant $\tilde{C}$ (which only depends on $T$ but not on $\tau$ ) such that

$$
\left(\frac{\alpha^{2}}{2}-C \alpha\right) \int_{\tau}^{T} e^{-\alpha Y_{s}}\left|Z_{s}\right|^{2} d s \leq \tilde{C}+\alpha \int_{\tau}^{T} e^{-\alpha Y_{s}} Z_{s} d W_{s}
$$

Taking conditional expectations on both sides of this inequality leads to

$$
\left(\frac{\alpha^{2}}{2}-C \alpha\right) \mathbb{E}\left[\int_{\tau}^{T} e^{-\alpha Y_{s}}\left|Z_{s}\right|^{2} d s \mid \mathcal{F}_{t}\right] \leq \tilde{C} \quad \mathbb{P} \text {-a.s. }
$$

which concludes the proof again by boundedness and $Y$ and by choosing $\alpha>2 C$.

\section{Acknowledgments}

We thank Kostas Kardaras and Michael Kupper for helpful comments and discussions. The authors acknowledge support from the DFG Research Center MATHEON.

\section{References}

[1] B. Acciaio, H. Föllmer, and I. Penner. Risk assessment for uncertain cash flows: model ambiguity, discounting ambiguity, and the role of bubbles. Finance Stoch., 16(4):669$709,2012$. 
[2] P. Artzner, F. Delbaen, J.-M. Eber, and D. Heath. Thinking coherently. RISK, 10:6871, 1997.

[3] P. Artzner, F. Delbaen, J.-M. Eber, and D. Heath. Coherent measures of risk. Math. Finance, 9(3):203-228, 1999.

[4] H. Assa. Lebesgue property of convex risk measures for bounded càdlàg processes. Methods Appl. Anal., 18(3):335-349, 2011.

[5] P. Barrieu and N. El Karoui. Pricing, hedging and optimally designing derivatives via minimization of risk measures. In Indifference pricing: Theory and applications, Princeton Series in Financial Engineering, pages 77-146, Princeton University Press, Princeton, NJ, 2009.

[6] E. Bayraktar, I. Karatzas, and S. Yao. Optimal stopping for dynamic convex risk measures. Illinois J. Math., 54(3):1025-1067, 2012.

[7] E. Bayraktar, C. Kardaras, and H. Xing. Valuation equations for stochastic volatility models. SIAM J. Financial Math., 3:351-373, 2012.

[8] E. Bayraktar and S. Yao. Optimal stopping for non-linear expectations - Part I Stochastic Process. Appl., 121(2):185-211, 2011.

[9] E. Bayraktar and S. Yao. Optimal stopping for non-linear expectations-Part II Stochastic Process. Appl., 121(2):212-264, 2011.

[10] K. Bichteler. Stochastic integration with jumps, volume 89 of Encyclopedia of Mathematics and its Applications. Cambridge University Press, Cambridge, 2002.

[11] P. Carr, T. Fisher, and J. Ruf. On the hedging of options on exploding exchange rates. Preprint, 2012.

[12] P. Cheridito, F. Delbaen, and M. Kupper. Coherent and convex monetary risk measures for bounded càdlàg processes. Stochastic Process. Appl., 112(1):1-22, 2004.

[13] P. Cheridito, F. Delbaen, and M. Kupper. Coherent and convex monetary risk measures for unbounded càdlàg processes. Finance Stoch., 9(3):369-387, 2005.

[14] P. Cheridito, F. Delbaen, and M. Kupper. Dynamic monetary risk measures for bounded discrete-time processes. Electron. J. Probab., 11:no. 3, 57-106, 2006.

[15] C. Dellacherie and P.-A. Meyer. Probabilities and potential, volume 29 of North-Holland Mathematics Studies. North-Holland Publishing Co., Amsterdam, 1978.

[16] C. Dellacherie and P.-A. Meyer. Probabilities and potential. B, volume 72 of NorthHolland Mathematics Studies. North-Holland Publishing Co., Amsterdam, 1982. Theory of martingales, Translated from the French by J. P. Wilson.

[17] N. El Karoui, C. Kapoudjian, E. Pardoux, S. Peng, and M. C. Quenez. Reflected solutions of backward SDE's, and related obstacle problems for PDE's. Ann. Probab., 25(2):702-737, 1997. 
[18] N. El Karoui and C. Ravanelli. Cash subadditive risk measures and interest rate ambiguity. Math. Finance, 19(4):561-590, 2009.

[19] H. Föllmer. The exit measure of a supermartingale. Z. Wahrscheinlichkeitstheorie und Verw. Gebiete, 21:154-166, 1972.

[20] H. Föllmer and A. Schied. Convex measures of risk and trading constraints. Finance Stoch., 6(4):429-447, 2002.

[21] H. Föllmer and A. Schied. Stochastic finance. Walter de Gruyter \& Co., Berlin, extended edition, 2011. An introduction in discrete time.

[22] M. Frittelli and E. Rosazza Gianin. Putting order in risk measures. Journal of Banking Finance, 26(7):1473-1486, 2002.

[23] S. Hamadène. Reflected BSDE's with discontinuous barrier and application. Stoch. Stoch. Rep., 74(3-4):571-596, 2002.

[24] K. Itô and S. Watanabe. Transformation of Markov processes by multiplicative functionals. Ann. Inst. Fourier (Grenoble), 15(fasc. 1):13-30, 1965.

[25] J. Jacod. Calcul stochastique et problèmes de martingales, volume 714 of Lecture Notes in Mathematics. Springer, Berlin, 1979.

[26] Ju. M. Kabanov, R. Š. Lipcer, and A. N. Širjaev. Absolute continuity and singularity of locally absolutely continuous probability distributions. I. Mat. Sb. (N.S.), 107(149)(3):364-415, 463, 1978.

[27] C. Kardaras. Numéraire-invariant preferences in financial modeling. Ann. Appl. Probab., 20(5):1697-1728, 2010.

[28] C. Kardaras, D. Kreher, and A. Nikeghbali. Strict local martingales and bubbles. Preprint, 2011.

[29] N. Kazamaki. Continuous exponential martingales and BMO, volume 1579 of Lecture Notes in Mathematics. Springer-Verlag, Berlin, 1994.

[30] M. Kobylanski. Backward stochastic differential equations and partial differential equations with quadratic growth. Ann. Probab., 28(2):558-602, 2000.

[31] J.-P. Lepeltier and M. Xu. Penalization method for reflected backward stochastic differential equations with one r.c.l.l. barrier. Statist. Probab. Lett., 75(1):58-66, 2005.

[32] M.-A. Morlais. Reflected backward stochastic differential equations and a class of non linear dynamic pricing rule. Stochastics An International Journal of Probability and Stochastic Processes, 85(1):1-26, 2013.

[33] J. Najnudel and A. Nikeghbali. A new kind of augmentation of filtrations. ESAIM Probab. Stat., 15(In honor of Marc Yor, suppl.):S39-S57, 2011. 
[34] K. R. Parthasarathy. Probability measures on metric spaces. Probability and Mathematical Statistics, No. 3. Academic Press Inc., New York, 1967.

[35] S. Peng. Nonlinear expectations, nonlinear evaluations and risk measures. In Stochastic methods in finance, volume 1856 of Lecture Notes in Math., pages 165-253. Springer, Berlin, 2004.

[36] S. Peng and M. Xu. The smallest $g$-supermartingale and reflected BSDE with single and double $L^{2}$ obstacles. Ann. Inst. H. Poincaré Probab. Statist., 41(3):605-630, 2005.

[37] D. Possamai and C. Zhou. Second order backward stochastic differential equations with quadratic growth. Preprint, 2012.

[38] F. Riedel. Optimal stopping under ambiguity in continuous time. Working paper 429, Institute of Mathematical Economics, Bielefeld university.

[39] F. Riedel. Optimal stopping with multiple priors. Econometrica, 77(3):857-908, 2009.

[40] E. Rosazza Gianin. Risk measures via g-expectations. Insurance Math. Econom., 39(1):19-34, 2006. 